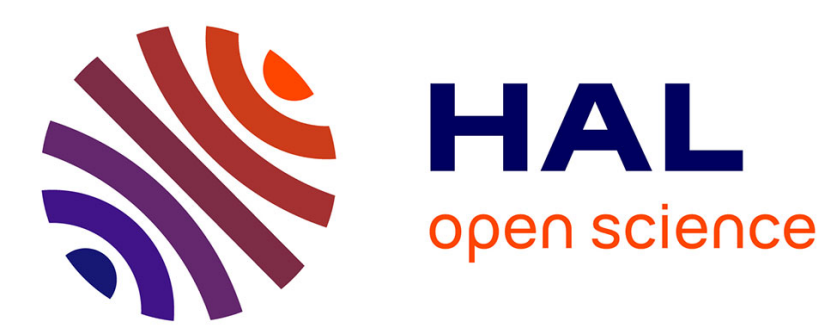

\title{
A CONSTITUTIVE INEQUALITY IN PLASTICITY INVOLVING LEFT-HAND AND RIGHT-HAND RATES
}

\author{
Ahmad Pouya
}

\section{- To cite this version:}

Ahmad Pouya. A CONSTITUTIVE INEQUALITY IN PLASTICITY INVOLVING LEFT-HAND AND RIGHT-HAND RATES. International Journal of Plasticity, 2004, 20, pp.1251-1279. hal00578029

\section{HAL Id: hal-00578029 https://hal.science/hal-00578029}

Submitted on 18 Mar 2011

HAL is a multi-disciplinary open access archive for the deposit and dissemination of scientific research documents, whether they are published or not. The documents may come from teaching and research institutions in France or abroad, or from public or private research centers.
L'archive ouverte pluridisciplinaire HAL, est destinée au dépôt et à la diffusion de documents scientifiques de niveau recherche, publiés ou non, émanant des établissements d'enseignement et de recherche français ou étrangers, des laboratoires publics ou privés. 
International Journal of Plasticity, 20 (2004) 1251-1279.

\title{
A CONSTITUTIVE INEQUALITY IN PLASTICITY INVOLVING LEFT-HAND AND RIGHT-HAND RATES
}

\author{
Ahmad POUYA \\ LCPC, 58 Bd Lefebvre, 75732 Paris Cedex 15 - France \\ ahmad.pouya@enpc.fr
}

\begin{abstract}
An incremental inequality for elastic-plastic materials involving right-hand (forward) and left-hand (backward) stress and strain rates is presented. This inequality is demonstrated as a theorem for standard elastic-plastic materials having a symmetric hardening matrix and also for generalized standard materials (Halphen and Nguyen 1975). It imposes some restrictions on the stress and strain rate discontinuities of these materials. Some applications of this inequality to deformation models of crystals and to micro-macro models are given. It is shown that this inequality generalizes some constitutive inequalities in plasticity and in particular the bi-incremental form of the normality flow rule (Hill 1967, 1968b). It includes and generalizes also some results of the Petryk (1989) inequality. Finally, it is shown that this inequality can be adopted as a postulate for elastic-plastic materials and that, in this case, it synthesizes the postulates of symmetry of the elastic compliant tensor, normality flow rule and, for models containing a hardening matrix and normal internal variables, the local symmetry of this matrix.
\end{abstract}

Keywords : B. Constitutive behaviour, Crystal plasticity, Elastic-plastic material, Inhomogeneous material, Polycrystalline material, Rate discontinuities

\section{Introduction}

Postulates describing the elastic-plastic behaviour of materials, as those proposed by Drucker $(1951,1959)$ and Iliushin $(1961)$, those reviewed by Hill $(1968 \mathrm{a}, \mathrm{b})$ and those proposed more recently by Rice (1971), Marigo (1989), Petryk (1989) and Zhou et al. (2003), can be divided in two families of global type and incremental type postulates. The first family involves integrals calculated along particular load paths whereas the second family involves the rates (derivative with respect to time) of stress, strain and of other state variables at a given state. For this last family of postulates it is important to distinguish between left-hand (or backward) and right-hand (or forward) rates of physical variables. Apart from any question of sign definition, left-hand $(l-h)$ and right-hand $(r-h)$ rates have 
different physical meanings. R-h rates for a given state correspond to processes beginning from this state, whereas 1-h rates correspond to processes ending to this state (Figure 1). In other words, $r$-h rates compare a present state to a neighbouring state which can be attained in the future, whereas 1-h rates compare the present state to a neighbouring state in the past from which the present state can have been obtained. For irreversible processes, which are not invariant to time reversing, $\mathrm{r}-\mathrm{h}$ and $\mathrm{l}-\mathrm{h}$ rates have naturally different properties. For plastic deformation process for instance, as it will be seen further, $r-h$ and $1-h$ rates of stress and strain admissible for a given state form different sets and have different properties (See the section 3.1 and the Figure 2). For instance, the classical theorem assuring the existence and uniqueness of a stress rate solution for a prescribed strain rate in perfect plasticity (Koiter 1960), is valid only for r-h rates.

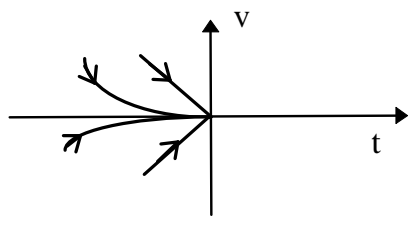

(a)

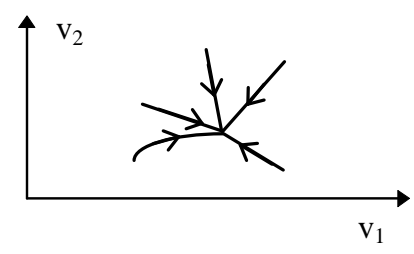

(c)

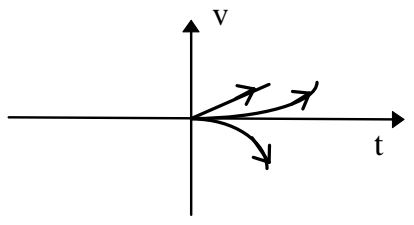

(b)

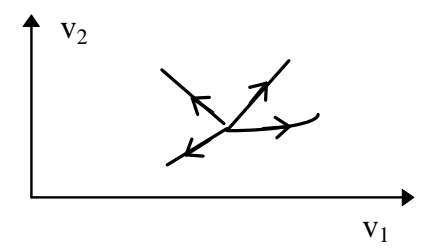

(d)

Figure 1: Left-hand rates for a given state correspond to processes ending to this state, whereas right-hand rates correspond to processes beginning from this state: left-hand rates (a) and right-hand rates (b) of a physical variable versus time, left-hand rates (c) and right-hand rates (b) in the plane of two physical variables $\left(\mathrm{v}_{1}, \mathrm{v}_{2}\right)$.

Most of the papers dealing with constitutive inequalities and incremental models in plasticity, except for Mandel (1965), consider exclusively r-h rates, even if this hypothesis is not always explicitly mentioned in the papers. This is the case for all the papers cited hereabove dealing with incremental type postulates of plasticity, and also those considering some extensions of previous works (Chen and Wang 2002, Cleja-Tigoiu 2003), and those dealing with modelling of different effects in plasticity (Chun et al. 2002, Chiarelli et al. 2003, Kang et al. 2003, Kaczmarek 2003, Zhang and Lee 2003, Stoughton 2002). This is also the case for papers dealing with more general frameworks than plasticity, for instance, rate- 
independent dissipative materials (Houlsby and Purzin 2000), damage processes (Taylor et al. 2002, Brünig 2002, 2003, Gupta and Burgström 2002, Naboulsi and Palazotto 2003), rate-dependent deformations (Henry and Haslach 2000, Gurtin 2003, Krempl and Khan 2003, Scheidler and Wrighte 2003, Haupt and Kersten 2003), and more general frameworks of dissipative processes (Ziegler 1983).

The choice of $r-h$ rates to describe the plastic behaviour is due obviously to the nature of problems more usually studied for elastic-plastic structures. Generally, in these problems, an initial state of the material or of the structure is given as well as a loading path and one is interested in the of evolution of this state under the prescribed loading, or in the prediction of the final state of the material or of the structure at the end of this evolution (Van Houtte et al. 2002, Yoshida and Uemori 2002, Langlois and Berveiller 2003, Li et al. 2003, Drozdov and Dorfmann 2003, Taleb and Sidoroff 2003). To study this problem by an incremental method, constitutive models involving $r-h$ increments are required. But let now consider the problem of rate discontinuities during different transformations. Stress and strain rates discontinuities can appear during deformation process of materials for different reasons. They can be due to discontinuities of the prescribed loading, for instance to abrupt changes in the load direction during cyclic tests. They can also be due to intrinsic properties of the materials. For instance, in crystals having multiple mechanisms of plastic deformation, the activation or inactivation of these mechanisms during a monotone loading path induce stress or strain rate discontinuities. Twinning transformation in crystals leads also to discontinuous deformation rates (Rajagopal and Srinivasa 1998a,b). Strain and strain rate discontinuities occur also during dynamic processes such as plastic wave propagation (Mandel 1965). The investigation of rate discontinuities deformation processes requires information about $1-h$ rates because rate discontinuity consist in the difference between $1-\mathrm{h}$ and $\mathrm{r}-\mathrm{h}$ rates at the same instant.

Another important family of problems requiring information about $1-\mathrm{h}$ rates are the retrograde problems. Let us consider the determination of the in situ state of a rock which has been disturbed during coring process from observations made on its state at laboratory, i.e., assuming that its present state is known. In this problem, the final state of the material is given as well as the loading path (stress modifications during the coring process) and one is interested in the initial state of the material. The incremental formulation of such a retrograde problem calls to $1-\mathrm{h}$ increments. The reconstruction of tectonic phenomena is another example of retrograde problems. Some methods of determination of residual stresses in materials in which the observation process itself modifies the stress distribution (for instance by cutting the sample in thin layers and observing the surface tractions by $\mathrm{X}$ rays), can also be classified as retrograde problems. These problems are usually very difficult to study because not always provided with (unique) solutions. They are very often studied indirectly and incompletely by using $r-h$ rates. Retrograde problems will not be studied in this paper, but we think that the constitutive inequality which will be presented can have interesting application to these problems. 
As mentioned above, the constitutive inequality which will be presented in this paper involves both $1-\mathrm{h}$ and $\mathrm{r}-\mathrm{h}$ stress and strain rates during deformation processes. This inequality will be introduced in the general framework of time independent dissipative materials. It will be demonstrated as a theorem for standard elastic-plastic materials with a symmetric hardening matrix (Mandel 1965, Hill 1966, Hill and Rice 1972) and also for generalized standard materials (Halphen and Nguyen 1975). Some consequences of this inequality on stress and strain rate discontinuities of materials will be given with examples of application to deformation models for crystals and to some micro-macro models. Then this inequality will be compared to other important constitutive inequalities or incremental type postulates in plasticity and the very general character of this inequality will be shown up. This leads naturally to examine the question of considering this inequality as a postulate. This question will be discussed in the final part of the paper.

\section{Notations}

Throughout this paper standard symbolic notation is used. Boldface lower case letters denote symmetric second-order tensors. Summation convention is adopted for repeated indices except for the index $n$. Fully contracted products are denoted by double dots $\left(\mathrm{a}_{\mathrm{ij}} \mathrm{b}_{\mathrm{ij}}=\mathbf{a}: \mathbf{b}\right.$, $\left.M_{\mathrm{ijkl}} \mathrm{a}_{\mathrm{kl}}=(\mathbb{M}: \mathbf{a})_{\mathrm{ij}}\right)$. Left-hand and right-hand derivatives of a piecewise differentiable function $\mathrm{f}$ of the (kinematic) time $\mathrm{t}$ at an instant $\mathrm{t}_{0}$ are denoted respectively by $\dot{\mathrm{f}}^{-}$and $\dot{\mathrm{f}}^{+}$and defined by the following definitions in which $\Delta \mathrm{t}$ is always positive :

$$
\dot{\mathrm{f}}^{-}\left(\mathrm{t}_{0}\right)=\operatorname{Lim}_{\Delta \mathrm{t} \rightarrow 0^{+}} \frac{\mathrm{f}\left(\mathrm{t}_{0}\right)-\mathrm{f}\left(\mathrm{t}_{0}-\Delta \mathrm{t}\right)}{\Delta \mathrm{t}} \quad, \quad \dot{\mathrm{f}}^{+}\left(\mathrm{t}_{0}\right)=\operatorname{Lim}_{\Delta \mathrm{t} \rightarrow 0^{+}} \frac{\mathrm{f}\left(\mathrm{t}_{0}+\Delta \mathrm{t}\right)-\mathrm{f}\left(\mathrm{t}_{0}\right)}{\Delta \mathrm{t}}
$$

\section{An inequality for time independent dissipative materials}

Let consider the quasistatic and isothermal transformation of a homogeneous time independent dissipative solid under applied external forces. Suppose that these forces induce uniform state of strains and stresses in the solid and that the material's behaviour can be fully characterized by the relations between these strains and stresses. Let $(\mathbf{e}, \mathbf{s})$ be a pair of conjugate tensors of strain and stress (Hill 1968a); i.e. such that the differential work of deformation is given by $\mathbf{s}$ :de. An equilibrium state can be characterized by the value of $\mathbf{e}$ and of some additional variables if the material is not elastic. Without specifying the physical nature of these variables and for the sake of simplicity, let suppose that they can be represented by a discrete set of scalar variables $(\xi \mathrm{k})$. An equilibrium state is then characterized by the values of $\mathbf{e}$ and $(\xi \mathrm{k})$, and the corresponding $\mathbf{s}$ is a function of these variables. This equilibrium state will be denoted by $(\mathbf{e}, \xi \mathrm{k} ; \mathbf{s})$.

During a quasistatic evolution, the material passes through successive equilibrium states. For a given equilibrium state $(\mathbf{e}, \xi \mathrm{k} ; \mathbf{s})$, one can consider two families of neighbouring equilibrium states. The first one consists of states $\left(\mathbf{e}+\delta \mathbf{e}, \xi k_{+}+\delta \xi \mathrm{k} ; \mathbf{s}+\delta \mathbf{s}\right)$ which can be attained in a close future by the material in consequence of a small variation of the external forces. These states will be called the right-hand neighbours of the state $(\mathbf{e}, \xi \mathrm{k} ; \mathbf{s})$. The 
second family consists of states $\left(\mathbf{e}-\delta \mathbf{e}^{\prime}, \xi^{\mathrm{k}}-\delta \xi^{\prime k} ; \mathbf{s}-\delta \mathbf{s}^{\prime}\right)$ of the material in a close past from which the present state $(\mathbf{e}, \xi \mathrm{k} ; \mathbf{s})$ can have been attained in consequence of a small variation of the external forces. Theses states will be called the left-hand neighbours of $(\mathbf{e}, \xi \mathrm{k} ; \mathbf{s})$. Because of the irreversible nature of the transformations, 1-h and r-h neighbours of a given state generally form two different sets.

Now consider a given state $(\mathbf{e}, \xi \mathrm{k} ; \mathbf{s})$. For some pairs of strain and stress increments $(\delta \mathbf{e}, \delta \mathbf{s})$, there exists at least one family of increments $\left(\delta \xi^{\mathrm{k}}\right)$ so that $\left(\mathbf{e}+\delta \mathbf{e}, \xi^{\mathrm{k}}+\delta \xi^{\mathrm{k}} ; \mathbf{s}+\delta \mathbf{s}\right)$ be a r-h neighbour of $(\mathbf{e}, \xi \mathrm{k} ; \mathbf{s})$, or in other words, so that $(\delta \mathbf{e}, \delta \xi \mathrm{k} ; \delta \mathbf{s})$ be a possible evolution beginning from $(\mathbf{e}, \xi \mathrm{k} ; \mathbf{s})$. These $(\delta \mathbf{e}, \delta \mathbf{s})$ pairs will be qualified as right-hand admissible pairs for the state $\left(\mathbf{e}, \xi^{\mathrm{k}} ; \mathbf{s}\right)$. In the same way, a pair of increments $\left(\delta \mathbf{e}^{\prime}, \delta \mathbf{s}^{\prime}\right)$ will be qualified as left-hand admissible for $(\mathbf{e}, \xi \mathrm{k} ; \mathbf{s})$ if there exists a family $\left(\delta \xi^{\prime} \mathrm{k}\right)$ so that $\left(\mathbf{e}-\delta \mathbf{e}^{\prime}, \xi \mathrm{k}_{-} \delta \xi^{\prime} \mathrm{k} ; \mathbf{s}-\right.$ $\left.\delta \mathbf{s}^{\prime}\right)$ be a 1 -h neighbour of $\left(\mathbf{e}, \xi^{\mathrm{k}} ; \mathbf{s}\right)$. Introducing a time parameter $\mathrm{t}$, a continuos and increasing function of the physical time, infinitesimal increments can be replaced by rates. A $\mathrm{r}$-h admissible pair of rates for a given state will be represented by $\left(\dot{\mathbf{e}}^{+}, \dot{\mathbf{s}}^{+}\right)$, and a 1-h admissible pair, by $\left(\dot{\boldsymbol{e}}^{-}, \dot{\mathbf{s}}^{-}\right)$. Notice the sign convention which is $(\delta \mathbf{e}, \delta \mathbf{s})=\left(\dot{\boldsymbol{e}}^{+} \delta \mathrm{t}, \dot{\mathbf{s}}^{+} \delta \mathrm{t}\right)$ and $\left(\delta \mathbf{e}^{\prime}, \delta \mathbf{s}^{\prime}\right)=\left(\dot{\boldsymbol{e}}^{-} \delta \mathrm{t}, \dot{\mathbf{s}}^{-} \delta \mathrm{t}\right)$ with $\delta \mathrm{t}>0$ in both cases. The sets of $\mathrm{r}-\mathrm{h}$ admissible pairs and of $1-\mathrm{h}$ admissible pairs for a given state $\left(\mathbf{e}, \xi^{\mathrm{k}} ; \mathbf{s}\right)$ will be denoted by $\boldsymbol{\Phi}^{+}$and $\boldsymbol{\Phi}^{-}$respectively. These sets characterize the incremental behaviour of the material in the neighbourhood of the state $(\mathbf{e}, \xi \mathrm{k} ; \mathbf{s})$.

Consider now a state $\left(\mathbf{e}, \xi^{\mathrm{k}} ; \mathbf{s}\right)$ and its relative sets $\boldsymbol{\Phi}^{+}$and $\boldsymbol{\Phi}^{-}$. Let $\left(\dot{\boldsymbol{e}}^{+}, \dot{\mathbf{s}}^{+}\right)$and $\left(\dot{\boldsymbol{e}}^{-}, \dot{\mathbf{s}}^{-}\right)$ denote two general elements of $\boldsymbol{\Phi}^{+}$and $\boldsymbol{\Phi}^{-}$respectively, or in other words, two pairs of rates respectively $\mathrm{r}-\mathrm{h}$ admissible and $\mathrm{l}-\mathrm{h}$ admissible for a same equilibrium state. The purpose of this paper is to discuss the following inequality involving these pairs of rates :

$$
\dot{\mathbf{s}}^{-}: \dot{\boldsymbol{e}}^{+}-\dot{\mathbf{s}}^{+}: \dot{\boldsymbol{e}}^{-} \geq 0
$$

First, we will show that this inequality holds as a theorem in the general framework of standard elastic-plastic behaviour.

\section{A theorem for elastic-plastic materials}

The general framework of standard elastic-plastic behaviour is considered here. In this framework and in the context of small strains, the elastic domain in the stress space is convex and delimited by a yield surface, and plastic strain rate verifies the normality flow rule (Hill 1950). Singular yield surfaces may be described by a number of yield functions $\mathrm{f}^{\mathrm{r}},(\mathrm{r}=1,2, \ldots)$, identified in some cases with the plastic potential of some physical deformation mechanisms (Budiansky and $\mathrm{Wu}$ 1962). To take account of the interaction between different mechanisms, Mandel (1965) and Hill (1966) introduced a hardening matrix $\mathrm{H}$, in which the element $\mathrm{H}^{\mathrm{kr}}$ represents the effect of the mechanism $\mathrm{r}$ on the hardening of the mechanism $\mathrm{k}$. For a prescribed strain rate, the existence of a stress rate solution is 
provided if $\mathrm{H}$ is symmetric and positive. In the context of finite strain, the convexity of the elastic domain is not measure invariant. In this case, the normality flow rule can be given by a bilinear differential expression involving work conjugate tensors and having the measure invariance property (Hill 1972). The earlier works of Koiter (1960), Mandel (1965) and Hill (1966) were extended to finite strain in different ways. In the model given by Hill and Rice (1972) a hardening matrix which is assumed to be symmetric appears explicitly. In the generalized standard materials defined by Halphen and Nguyen (1975) neither a hardening matrix nor symmetry hypothesis appear explicitly, but the formulation in terms of generalized potentials, assumes implicitly some symmetry properties. These two models of very general character will be considered in the following for the demonstration of the inequality (1) as a theorem.

But let us first see more precisely what is the difference between the 1-h and $\mathrm{r}-\mathrm{h}$ rate properties in standard elastic-plastic behaviour.

\subsection{Differences between left-hand and right-hand rates in plasticity}

For irreversible processes in general and for plastic deformation in particular, $1-\mathrm{h}$ and $\mathrm{r}-\mathrm{h}$ rates have different properties. Let us illustrate theses differences in plasticity by considering the simple case of a one-dimensional perfect elastic-plastic model. The elastic domain is defined by $|\sigma| \leq \sigma_{\mathrm{y}}$ where $\sigma_{\mathrm{y}}$ is a positive constant, and let the elastic behaviour be linear with a modulus E. Consider now a state of the material with a strain $\varepsilon_{0}$ and a stress $\sigma=\sigma_{\mathrm{y}}$ (Figure 2). For this state, a r-h. stress rate is admissible (compatible with the condition $\forall \mathrm{t}$; $\mid$ $\left.\sigma(\mathrm{t}) \mid \leq \sigma_{\mathrm{y}}\right)$ only if $\dot{\sigma}^{+} \leq 0$, whereas a 1 -h. stress rate is admissible only if $\dot{\sigma}^{-} \geq 0$. The normality flow rule at this state can be written as $\dot{\varepsilon}^{\mathrm{p}+} \geq 0$ and $\dot{\varepsilon}^{\mathrm{p}} \geq 0$. Now, let a total strain rate be given and try to find the stress rate solution. First consider the case of r-h rates and denote the prescribed strain rate by $\dot{\varepsilon}^{+}$. If $\dot{\varepsilon}^{+} \geq 0$, then the solution is $\left(\dot{\sigma}^{+}=0, \dot{\varepsilon}^{\mathrm{p}+}=\dot{\varepsilon}^{+} \geq 0\right)$, and if $\dot{\varepsilon}^{+}<0$ then the solution is $\left(\dot{\sigma}^{+}=\mathrm{E} \dot{\varepsilon}^{+}<0\right.$, $\left.\dot{\varepsilon}^{\mathrm{p}+}=0\right)$. So, for each value of $\dot{\varepsilon}^{+}$a unique solution of stress rate exist. Now consider the case of 1-h rates and let denote the prescribed strain rate by $\dot{\varepsilon}^{-}$. If $\dot{\varepsilon}^{-}>0$ then there are two possible solutions for the stress and plastic strain rates : $\left(\dot{\sigma}^{-}=\mathrm{E} \dot{\varepsilon}^{-}, \dot{\varepsilon}^{\mathrm{p}-}=0\right)$ corresponding to an elastic path, and $\left(\dot{\sigma}^{-}=0, \dot{\varepsilon}^{\mathrm{p}^{-}}=\dot{\varepsilon}^{-}\right)$ corresponding to a pure plastic path. If $\dot{\varepsilon}^{-}=0$ then there is a unique solution $\left(\dot{\sigma}^{-}=0, \dot{\varepsilon}^{\mathrm{p}-}=0\right)$. If $\dot{\varepsilon}^{-}<0$, then there is no solution verifying the condition of admissibility of the stress rate, $\dot{\sigma}^{-} \geq$ 0 , the 1 -h normality flow rule, $\dot{\varepsilon}^{\mathrm{p}^{-}}=\dot{\varepsilon}^{-}-\mathrm{E}^{-1} \dot{\sigma}^{-} \geq 0$. So, depending on the value of $\dot{\varepsilon}^{-}$, a solution of stress rate can exist or not, and when this solution exists, it can be not unique. 


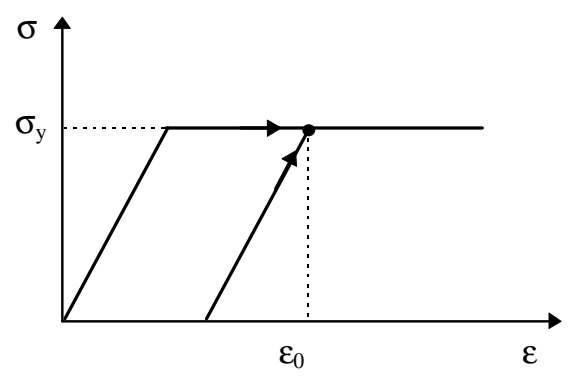

(a)

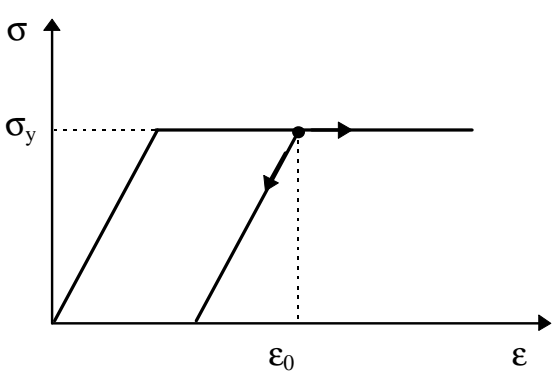

(b)

Figure 2. Admissible left-hand rates (a) and right-hand rates (b) in perfect plasticity

Therefore, the fundamental theorem ensuring in perfect plasticity the existence and uniqueness of a stress rate solution for a prescribed strain rate (Koiter 1960) is not valid for 1 -h rates! This theorem is only valid for $r-h$ rates. This shows the radical differences between 1-h and $\mathrm{r}$-h rates. Theses differences appear in the physical models describing theses rates, here the flow rules, and have their origin in the irreversible nature of the phenomena described by these models.

\subsection{Models with a hardening matrix}

The general equations of these models, as given by Hill and Rice (1972) for finite strain, are the followings :

$$
\begin{aligned}
& \dot{\mathbf{e}}=\mathbb{M}: \dot{\mathbf{s}}+\dot{\boldsymbol{e}}^{\mathrm{p}} \\
& \dot{\mathbf{e}}^{\mathrm{p}}=\dot{\gamma}^{\mathrm{k}} \partial \mathrm{f}^{\mathrm{k}} / \partial \mathbf{s}
\end{aligned}
$$

In these equations $(\mathbf{e}, \mathbf{s})$ represents a pair of conjugate tensors of strain and stress, $\dot{\mathbf{e}}^{\mathrm{p}}$ is the plastic strain rate and $\mathbb{M}$ is the fourth order tensor of elastic compliance with the usual symmetries $M_{\mathrm{ijkl}}=M_{\mathrm{jikl}}=M_{\mathrm{klij}}$. The yield function $\mathrm{f}^{\mathrm{k}}$ is a convex function of $\mathbf{s}$, depending on some parameters $\gamma^{\mathrm{k}}$. It is assumed to be at least $\mathrm{C}^{1}$ continuous with respect to $s$ and $\gamma^{\mathrm{k}}$, and restricted to remain always negative or zero :

$$
\forall \mathrm{t} ; \quad \mathrm{f}^{\mathrm{k}} \leq 0
$$

The parameters $\gamma^{\mathrm{k}}$ are defined as integrals of the rates $\dot{\gamma}^{\mathrm{k}}$, given themselves by the following flow rules (normality rule) :

$$
\forall \mathrm{t} ; \quad \begin{aligned}
& \dot{\gamma}^{\mathrm{k}} \geq 0 \\
& \dot{\gamma}^{\mathrm{k}}=0 \text { if } \mathrm{f}^{\mathrm{k}}<0 \text { or if } \mathrm{f}^{\mathrm{k}}=0 \text { and } \dot{\mathrm{f}}^{\mathrm{k}}<0
\end{aligned}
$$

where : 
with :

$$
\begin{gathered}
\dot{\mathrm{f}}^{\mathrm{k}}=\left(\partial \mathrm{f}^{\mathrm{k}} / \partial \mathbf{s}\right): \dot{\mathbf{s}}-\mathrm{H}^{\mathrm{kl}} \dot{\gamma}^{1} \\
\mathrm{H}^{\mathrm{kl}} \equiv-\partial \mathrm{f}^{\mathrm{k}} / \partial \gamma^{\mathrm{l}}
\end{gathered}
$$

These flow rules define in fact $\mathrm{r}-\mathrm{h}$ rates, and because $\mathrm{r}-\mathrm{h}$ and $\mathrm{l}-\mathrm{h}$ rates must be rigorously distinguished in this paper, the above $r$ - $h$ flow rules will be rewritten as :

$$
\forall \mathrm{t} ; \quad \begin{aligned}
& \dot{\gamma}^{+\mathrm{k}} \geq 0 \\
& \dot{\gamma}^{+\mathrm{k}}=0 \text { if } \mathrm{f}^{\mathrm{k}}<0 \text { or if } \mathrm{f}^{\mathrm{k}}=0 \text { and } \dot{\mathrm{f}}^{+\mathrm{k}}<0
\end{aligned}
$$

The $l$-h flow rules allowing the condition (4) to be verified, are the followings (Mandel 1965):

$$
\forall \mathrm{t} ; \quad \begin{aligned}
& \dot{\gamma}^{-\mathrm{k}} \geq 0 \\
& \dot{\gamma}^{-\mathrm{k}}=0 \text { if } \mathrm{f}^{\mathrm{k}}<0 \text { or if } \mathrm{f}^{\mathrm{k}}=0 \text { and } \dot{\mathrm{f}}^{-\mathrm{k}}>0
\end{aligned}
$$

In general, relations involving equalities are the same for $1-\mathrm{h}$ and $\mathrm{r}-\mathrm{h}$ rates, but those involving inequalities are different. For instance, the equality (5) can be written as $\dot{\mathrm{f}}^{-\mathrm{k}}=\left(\partial \mathrm{f}^{\mathrm{k}} /\right.$ $\partial \mathbf{s}): \dot{\mathbf{s}}^{-}-\mathrm{H}^{\mathrm{kl}} \dot{\gamma}^{-1}$ and $\dot{\mathrm{f}}^{+\mathrm{k}}=\left(\partial \mathrm{f}^{\mathrm{k}} / \partial \mathbf{s}\right): \dot{\mathbf{s}}^{+}-\mathrm{H}^{\mathrm{kl}} \dot{\gamma}^{+1}$. On the other hand, it may be noticed that $\partial \mathrm{f}^{\mathrm{k}} /$ $\partial \mathbf{s}$ and $\mathrm{H}^{\mathrm{kl}}$ are continuos functions of time since $\mathrm{f}^{\mathrm{k}}$ is supposed to be $\mathrm{C}^{1}$ continuos with respect to $\mathbf{s}$ and $\gamma^{\mathrm{k}}$ and these variables are assumed to be piecewise differentiable and so continuous with respect to time. Therefore, the discontinuities of $\dot{\mathrm{f}}^{\mathrm{k}}$ only stem from discontinuities of $\dot{\boldsymbol{s}}$ and $\dot{\gamma}^{1}$.

Now consider a general equilibrium state and two pairs of strain and stress rates, $\left(\dot{\mathbf{e}}^{-}, \dot{\mathbf{s}}^{-}\right)$ and $\left(\dot{\mathbf{e}}^{+}, \dot{\mathbf{s}}^{+}\right)$, respectively l-h and r-h admissible for this state. For each one of these pairs there exists a family (not necessarily unique) of rates $\left(\dot{\gamma}^{\mathrm{k}}, \dot{\mathrm{f}}^{\mathrm{k}}\right)$ satisfying the equations (2),(3),(5) and the corresponding flow rules (6) or (7). Let denote by $\left(\dot{\gamma}^{-k}, \dot{\mathrm{f}}^{-\mathrm{k}}\right)$ such a family for the first pair and by $\left(\dot{\gamma}^{+k}, \dot{f}+k\right)$ such a family for the second pair. Then, let define the following quantity for a general index $n$ (without summation on $n$ ) :

$$
\Delta_{\mathrm{n}} \equiv \dot{\mathrm{f}}^{\mathrm{n}-} \dot{\gamma}^{\mathrm{n}+}-\dot{\mathrm{f}}^{\mathrm{n}+} \dot{\gamma}^{\mathrm{n}-}
$$

and try to determine the sign of this quantity. For the given state, two cases may be considered for the yield function $\mathrm{f}^{\mathrm{n}}$ : it can be negative or zero. Let examine these two cases separately :

- If $\mathrm{f}^{\mathrm{n}}<0$, then the continuity of $\mathrm{f}^{\mathrm{n}}$ with respect to time implies $\mathrm{f}^{\mathrm{n}}<0$ in a vicinity of the considered instant or state. So at this instant (or at the considered state) $\dot{\gamma}^{\mathrm{n}-}=0$ and $\dot{\gamma}^{\mathrm{n}+}=0$ and hence $\Delta_{\mathrm{n}}=0$.

- If $\mathrm{f}^{\mathrm{n}}=0$, the condition $\mathrm{f}^{\mathrm{n}} \leq 0$ implies that $\dot{\mathrm{f}}^{\mathrm{n}-} \geq 0$ and $\dot{\mathrm{f}}^{\mathrm{n}+} \leq 0$. Then, due to the positive values of $\dot{\gamma}^{\mathrm{n}-}$ and $\dot{\gamma}^{\mathrm{n}+}$, we have $\Delta_{\mathrm{n}} \geq 0$.

So, in all cases, $\Delta_{\mathrm{n}}$ is non negative :

$$
\Delta_{\mathrm{n}} \geq 0
$$

This property is a direct consequence of the flow rules. Now, substituting $\dot{\mathrm{f}}^{\mathrm{n}-}$ and $\dot{\mathrm{f}}^{\mathrm{n}+}$ in $\Delta_{\mathrm{n}}$ by their expressions given by (5), one finds : 


$$
0 \leq \Delta_{\mathrm{n}}=\dot{\gamma}^{\mathrm{n}+}\left(\partial \mathrm{f}^{\mathrm{n}} / \partial \mathbf{s}\right): \dot{\mathbf{s}}^{-}-\dot{\gamma}^{\mathrm{n}-}\left(\partial \mathrm{f}^{\mathrm{n}} / \partial \mathbf{s}\right): \dot{\mathbf{s}}^{+}-\dot{\gamma}^{\mathrm{n}+} \mathrm{H}^{\mathrm{ns}} \dot{\gamma}^{\mathrm{s}-}+\dot{\gamma}^{\mathrm{n}-} \mathrm{H}^{\mathrm{ns}} \dot{\gamma}^{\mathrm{s}+}
$$

Summation of this inequality upon $\mathrm{n}$ gives:

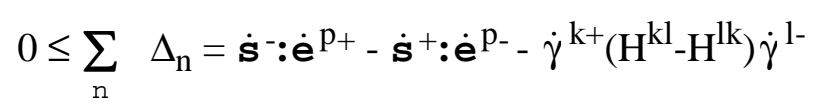

The contribution of $\mathrm{H}$ terms to the second member of (8) vanishes because of the symmetry of the matrix $\mathrm{H}$, and one finds :

$$
\dot{\mathbf{s}}^{-}: \dot{\boldsymbol{e}}^{\mathrm{p}_{+}}-\dot{\mathbf{s}}^{+}: \dot{\boldsymbol{e}}^{\mathrm{p}_{-}} \geq 0
$$

This fundamental inequality is thus a consequence only of the normality flow rule and of the local symmetry of the hardening matrix. Moreover, the diagonal symmetry of the elastic compliance tensor $M$ provides the following equality :

$$
\dot{\mathbf{s}}^{-}: \dot{\mathrm{e}}^{\mathrm{e}_{+}}-\dot{\mathbf{s}}^{+}: \dot{\mathrm{e}}^{\mathrm{e}_{-}}=0
$$

The addition of (9) and (10) provides then :

$$
\dot{\mathbf{s}}^{-}: \dot{\mathbf{e}}^{+}-\dot{\mathbf{s}}^{+}: \dot{\boldsymbol{e}}^{-} \geq 0
$$

The inequality (1) is so demonstrated.

It may be noticed that this inequality is measure invariant (Hill 1968a, 1968b, 1972) because it involves conjugated stress and strain tensors.

This was given in a less general framework (diagonal hardening matrix) by Pouya (1991), and then by Pouya (1993) for the study of rates discontinuities (see further in this paper).

\subsection{Generalized standard materials}

The generalized standard material (Halphen et al., 1975) is defined by a specific free energy $\varphi\left(\mathbf{e}^{\mathrm{e}}, \alpha^{\mathrm{k}}\right)$ where $\mathbf{e}^{\mathrm{e}}$ is the elastic strain and $\left(\alpha^{\mathrm{k}}\right)$ a family of internal variables. The stress $\mathbf{s}$ and the thermodynamic force $\mathrm{A}^{\mathrm{k}}$ associated with $\alpha^{\mathrm{k}}$ are given by :

$$
\mathbf{s}=\partial \varphi / \partial \mathbf{e}^{\mathrm{e}}, \mathrm{A}^{\mathrm{k}}=\partial \varphi / \partial \alpha^{\mathrm{k}}
$$

It is assumed that there exists a set of potentials $\mathrm{f}^{\mathrm{r}}\left(\mathbf{s}, \mathrm{A}^{\mathrm{k}}\right)$, convex and differentiable in $\mathbf{s}$ and $\mathrm{A}^{\mathrm{k}}$ and restricted to remain always negative or zero : $\mathrm{f}^{\mathrm{r}} \leq 0$. The plastic strain rate $\dot{\mathrm{e}}^{\mathrm{p}}$ and $\dot{\alpha}^{\mathrm{k}}$ are given by :

$$
\left(\dot{\boldsymbol{e}}^{\mathrm{p}}, \dot{\alpha}^{\mathrm{k}}\right)=\lambda^{\mathrm{r}}\left(\partial \mathrm{f}^{\mathrm{r}} / \partial \mathbf{s}, \partial \mathrm{f}^{\mathrm{r}} / \partial \mathrm{A}^{\mathrm{k}}\right)
$$

where the following generalized normality rules are supposed to hold (for right-hand rates) :

$$
\begin{aligned}
& \forall \mathrm{t} ; \quad \lambda^{\mathrm{r}} \geq 0 \\
& \lambda^{\mathrm{r}}=0 \text { if } \mathrm{f}^{\mathrm{r}}<0 \quad \text { or if } \mathrm{f}^{\mathrm{r}}=0 \text { and } \dot{\mathrm{f}}^{\mathrm{r}}<0
\end{aligned}
$$

with : 


$$
\dot{\mathrm{f}}^{\mathrm{r}}=\left(\partial \mathrm{f}^{\mathrm{r}} / \partial \mathbf{s}\right): \dot{\mathbf{s}}+\left(\partial \mathrm{f}^{\mathrm{r}} / \partial \mathrm{A}^{\mathrm{k}}\right) \dot{\mathrm{A}}^{\mathrm{k}}
$$

Denoting in a concise form $\mathbf{A}=\left(\mathbf{s}, \mathrm{A}^{\mathrm{k}}\right)$ and $\boldsymbol{\alpha}=\left(\mathbf{e}^{\mathrm{p}},-\alpha^{\mathrm{k}}\right)$, one can notice that the generalized standard material behaves as a perfectly plastic material with respect to the generalized stress $\mathbf{A}$ and the generalized plastic strain $\boldsymbol{\alpha}$.

Obviously, for the condition $\mathrm{f}^{\mathrm{r}} \leq 0$ to hold, the last inequality in (13) must be changed into $\dot{\mathrm{f}}^{\mathrm{r}-}>0$ for left-hand rates. One can then define, as in the case of models with a hardening matrix, the quantity $\Delta_{n}=\lambda^{n+} \dot{\mathrm{f}}^{\mathrm{n}-}-\lambda^{\mathrm{n}-} \dot{\mathrm{f}}^{\mathrm{n}+}$ and show that it takes always positive values. Then, after summation upon $n$, one finds :

$$
\dot{\mathbf{A}}^{-} \cdot \dot{\boldsymbol{\alpha}}^{+}-\dot{\mathbf{A}}^{+} \cdot \dot{\boldsymbol{\alpha}}^{-} \geq 0
$$

where (.) represents the scalar product $\dot{\mathbf{A}} \cdot \dot{\alpha} \equiv \dot{\mathbf{s}}: \dot{\mathrm{e}}^{\mathrm{p}}+\dot{\mathrm{A}}^{\mathrm{k}} \dot{\alpha}^{\mathrm{k}}$. This inequality is the generalized form of (9).

Substituting $\mathbf{A}$ and $\boldsymbol{\alpha}$ in (14) by their expressions in terms of $\mathbf{s}, \mathbf{A}, \mathbf{e}^{\mathrm{p}}$ and $\alpha^{\mathrm{k}}$, one finds :

$$
\dot{\mathbf{s}}^{-}: \dot{\boldsymbol{e}}^{\mathrm{p}+}-\dot{\mathbf{s}}^{+}: \dot{\mathbf{e}}^{\mathrm{p}-}-\dot{\mathrm{A}}^{\mathrm{k}_{-}} \dot{\alpha}^{\mathrm{k}_{+}}+\dot{\mathrm{A}}^{\mathrm{k}_{+}} \dot{\alpha}^{\mathrm{k}_{-}} \geq 0
$$

The definition (11) and the symmetries of the second order cross derivatives of $\varphi\left(\mathbf{e}^{\mathrm{e}}, \alpha^{\mathrm{k}}\right)$ provide :

and

$$
-\dot{\mathrm{A}}^{\mathrm{k}_{-}} \dot{\alpha}^{\mathrm{k}_{+}}+\dot{\mathrm{A}}^{\mathrm{k}_{+}} \dot{\alpha}^{\mathrm{k}_{-}}=0
$$

$$
\dot{\boldsymbol{s}}^{-}: \dot{\mathrm{e}}^{\mathrm{e}+}-\dot{\boldsymbol{s}}^{+}: \dot{\mathrm{e}}^{\mathrm{e}-}=0
$$

Substitution of (16) in (15) then provides (9). Adding the equality (17) to (9), one finds (1).

The inequality (1) is demonstrated in this way for the generalized standard materials.

\subsection{Hypothesis of symmetry of the hardening matrix}

One may notice that the full symmetry of $\mathrm{H}$ is not required to demonstrate (9). In fact, if two mechanisms $\mathrm{k}$ and $\mathrm{l}$ can not be simultaneously potentially active, then every time for one at least of these mechanisms the yield function is negative and so, for this mechanism $\dot{\gamma}^{+}$and $\dot{\gamma}^{-}$are equal to zero. This implies that for this pair of mechanisms $\dot{\gamma}^{\mathrm{k}+} \dot{\gamma}^{1-}=\dot{\gamma}^{1+} \dot{\gamma}^{\mathrm{k}-}=0$ at every instant, and so the contributions of $\mathrm{H}^{\mathrm{kl}}$ and $\mathrm{H}^{\mathrm{lk}}$ to the sum (8) vanish.

This leads as to consider, as a necessary condition for the inequality (1) to be valid, a weaker symmetry property of the hardening matrix which will be called the local symmetry. To describe this property, let us consider a material with a family of yield functions $\mathrm{f} r$. In general, not all pairs of these functions can vanish simultaneously. For instance, in the context of small strains and for the Tresca material, the elastic domain is a cylinder with a hexagonal section in the principal stresses space (Figure 3). This domain is described by six 
regular yield functions each one corresponding to one face of the cylinder. In this case, the yield functions corresponding to two parallel faces can not vanish simultaneously.

In the same way, in the case of crystals with different plastic deformation mechanisms, described by yield function $\mathrm{f}^{\mathrm{r}}$, in general not all mechanisms can be potentially active at the same time (the mechanism $\mathrm{r}$ is said to be potentially active when $\mathrm{f}^{\mathrm{r}}=0$ ). Then, among the non-diagonal terms of the hardening matrix, one can distinguish between those corresponding to pairs of mechanisms which can be potentially active at the same time and those corresponding to pairs which can not. The requirement is that the hardening matrix must be symmetric over all pairs of mechanisms which can be potentially active simultaneously. In this case the hardening matrix will be called locally symmetric. Of course, a symmetric hardening matrix is also locally symmetric. The local symmetry property is weaker than the (full) symmetry of hardening matrix and is the only condition required for the demonstrations given here above. It is interesting to notice that only this weaker property of local symmetry is required also for demonstrations given in Mandel (1965) and Hill (1966) even if the distinction between the two types of symmetries has not been made in these papers. Otherwise, if the hardening model describes only the behaviour in the vicinity of a (singular) point of the yield surface (i.e., if it has a local character), as it is the case for the Hill and Rice (1972) model, then all the yield functions considered in the model can vanish simultaneously. In this case, local symmetry of the hardening matrix is equivalent to its (full) symmetry.

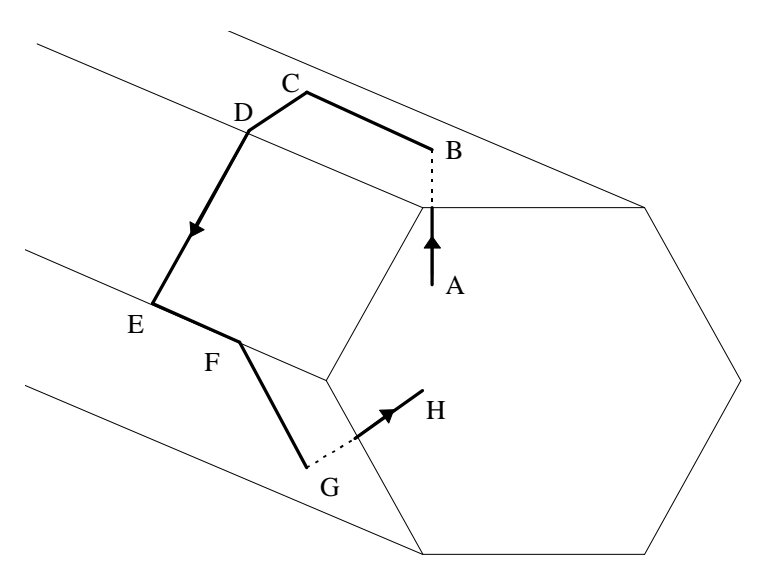

Figure 3: Stress rate discontinuities appear during a loading path when the load direction changes (point $\mathrm{C}$ ), or when the stress point on the yield surface crosses singularities (point $\mathrm{D}$ ) or when the singularity type or the deformation mode change (points E,F, B and G). 


\section{Consequences of the constitutive inequality for rate discontinuities}

\subsection{Stress and strain rate discontinuities}

The evolution of stress and strain tensors along loading paths can include rate discontinuities at some singular points. These discontinuities can be due directly to rate discontinuities of the prescribed load. An abrupt change of the load direction, during fatigue cyclic tests for instance, generates stress and strain rates discontinuities. The discontinuities can result also from intrinsic properties of the material. For instance, for multipotential elastic-plastic materials, stress rate discontinuities may appear during a straining path with a constant strain rate due in a general way to changes in the family of active mechanisms. In this case, from a geometric point of view, discontinuities appear when the representative stress point crosses singularities of the yield surface or when the singularity type changes. For instance, let consider the example of the Tresca solid for which the yield surface is a cylinder with a hexagonal section in principal stresses space (Figure 3). Discontinuities appear when the stress point attains or leaves the yield surface from within the elastic domain (points B and G), attains or leaves an edge by a path inscribed in a face (points E and $\mathrm{F}$ ), crosses an edge (point D), changes direction in a face (point C), etc. At all these points the inequality (1) remains valid.

Consider now a loading path and let denote by $\left(\dot{\mathbf{e}}^{-}, \dot{\mathbf{s}}^{-}\right)$and $\left(\dot{\mathbf{e}}^{+}, \dot{\mathbf{s}}^{+}\right)$respectively the real l-hand and r-h pairs of rates the material undergoes during this path. Because real $1-\mathrm{h}$ and $\mathrm{r}-\mathrm{h}$ pairs are necessarily $1-h$ and $r-h$ admissible respectively, the inequality (1) can be written for $\left(\dot{\mathbf{e}}^{-}, \dot{\mathbf{s}}^{-}\right)$and $\left(\dot{\mathbf{e}}^{+}, \dot{\mathbf{s}}^{+}\right)$. Let consider now the case of a straining path with a continuous prescribed strain rate $\dot{\mathbf{e}}$, i.e. so that at every instant, $\dot{\mathbf{e}}^{+}=\dot{\mathbf{e}}^{-}=\dot{\mathbf{e}}$. In this case, the inequality (1) leads to $\left(\dot{\mathbf{s}}^{+}-\dot{\mathbf{s}}^{-}\right): \dot{\mathbf{e}} \leq 0$. Denoting by $[[\dot{\mathbf{s}}]]$ the discontinuity $\left(\dot{\mathbf{s}}^{+}-\dot{\mathbf{s}}^{-}\right)$, one finds :

$$
[[\dot{\mathbf{s}}]]: \dot{e} \leq 0
$$

For a loading path with a continuous prescribed stress rate, i.e. $\dot{\mathbf{s}}^{+}=\dot{\mathbf{s}}^{-}=\dot{\mathbf{s}}$ at every instant, the inequality (1) leads to the following inequality in which $[[\dot{\mathbf{e}}]]=\dot{\mathbf{e}}^{+}-\dot{\mathbf{e}}^{-}$:

$$
[[\dot{\mathbf{e}}]]: \dot{\mathbf{s}} \geq 0
$$

These inequalities impose some restrictions on the phenomenological form of strainstress curves. In fact, let consider a proportional straining path for which the prescribed strain is given by $\mathbf{e}=\mathrm{p} \boldsymbol{\psi}$ where $\mathrm{p}$ is a scalar monotonic and differentiable function of time, and where the strain direction $\psi$ is a constant second order tensor normalized to unity $(\psi: \psi=1)$. Let denote by $q=\mathbf{s}: \psi$, the stress component in the load direction. If the strain rate is continuous, i.e., if $\dot{\mathrm{p}}^{+}=\dot{\mathrm{p}}^{-}$then the inequality (18) implies :

$$
[[\mathrm{dq} / \mathrm{dp}]] \leq 0
$$


This inequality can be interpreted by saying that "the intrinsic slope discontinuities of the stress-strain curve are negative", where intrinsic means "not due to rate discontinuity of the applied load".

This result is illustrated by the points A,B,C,E and F on the figure (4) (the points on this figure are independent of those of the figure 3 ). The dotted lines on this figure are forbidden by the inequality (20). The points D and G correspond to load direction changing $\left(\dot{\mathrm{p}}^{+}=-\dot{\mathrm{p}}^{-}\right.$, $\psi$ remaining constant). For these points, the inequality (1) leads to [[dq/dp]] $\geq 0$.

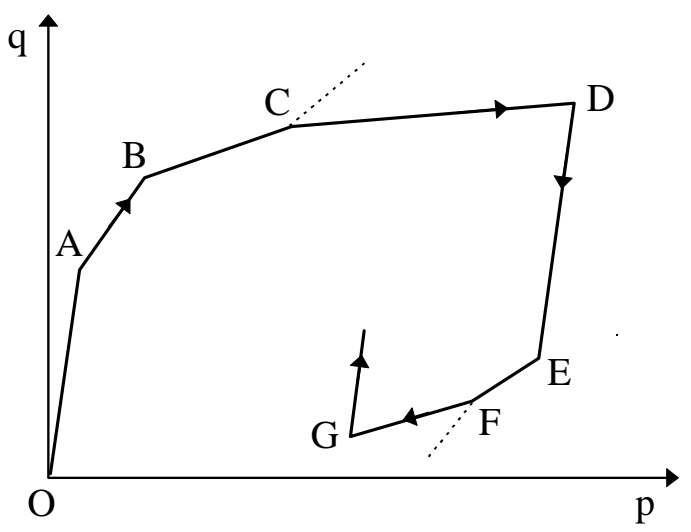

Figure 4 - Intrinsic slope discontinuities of the hardening curve of multipotential materials with symmetric hardening matrix are negative. The dotted lines on this figure are forbidden for these materials.

The slope variation sign at the point A (the end of the elastic stage) as well as at the points $\mathrm{D}$ and $\mathrm{G}$ (supposing that the stage immediately following the change in the loading direction is elastic) is due to the normality rule only. But to obtain the result concerning the intermediate points $\mathrm{B}, \mathrm{C}, \mathrm{E}$ and $\mathrm{F}$, the symmetry of the hardening matrix, or at least its local symmetry, is required.

\subsection{Application to deformation models of crystals}

In this section, we will show that the results obtained here above for rate discontinuities impose some limitations on phenomenological modelling of the single crystalline behaviour with models presented in the section 3.2. The framework of small strain theory is considered in this section.

In fact, stress-strain curves of single crystals show different stages of hardening corresponding to different slopes and features. According to microscopic observations, these stages correspond to the activity of different mechanisms of deformation. Let us consider the problem of phenomenological modelling of single crystalline elastic-plastic behaviour when 
plastic strain is produced by slip on different crystallographic planes. The plastic strain can be written as :

$$
\dot{\boldsymbol{\varepsilon}}^{\mathrm{P}}=\sum_{\mathrm{k}} \dot{\gamma}^{\mathrm{k}} \mathbf{a}^{\mathrm{k}}
$$

where $\dot{\gamma}^{\mathrm{k}}$ represents the slip rate on $k$ th system and the matrix $\mathbf{a}^{\mathrm{k}}$ represents the strain direction for slip on this system. This matrix is given by :

$$
\mathbf{a}^{\mathrm{k}}=\frac{1}{2}\left(\overrightarrow{\mathrm{m}}^{\mathrm{k}} \otimes \overrightarrow{\mathrm{n}}^{\mathrm{k}}+\overrightarrow{\mathrm{n}}^{\mathrm{k}} \otimes \overrightarrow{\mathrm{m}}^{\mathrm{k}}\right)
$$

where the unit vectors $\overrightarrow{\mathrm{m}}^{\mathrm{k}}$ and $\overrightarrow{\mathrm{n}}^{\mathrm{k}}$ represent the slip plane and slip direction respectively. The slip rate $\dot{\gamma}^{\mathrm{k}}$ verifies the standard flow rules (6) or (7) with respect to the plastic potential $\mathrm{f}^{\mathrm{k}}$ defined as :

$$
\mathrm{f}^{\mathrm{k}}=\tau^{\mathrm{k}}-\tau_{\mathrm{c}}^{\mathrm{k}}
$$

In this expression, $\tau^{\mathrm{k}}$ is the resolved shear stress on the $k$ th slip system and is given by $\tau^{\mathrm{k}}$ $=\boldsymbol{\sigma}: \mathbf{a}^{\mathrm{k}}$, and $\tau_{\mathrm{c}}$ is the critical shear stress on this system. The variation of the critical shear stress with deformation is given by the hardening matrix defined as :

$$
\mathrm{H}^{\mathrm{kl}}=\partial \tau_{\mathrm{c}} / \partial \gamma^{1}
$$

Now, let assume that a single crystal is submitted to a monotonous proportional straining path given by :

$$
\varepsilon=\mathrm{p} \psi
$$

where $\mathrm{p}$ is a positive increasing function of time and $\psi$ is a constant symmetric matrix. The elastic strain rate is given by :

$$
\dot{\boldsymbol{\varepsilon}}^{\mathrm{e}}=\dot{\mathrm{p}} \boldsymbol{\psi}-\sum_{\mathrm{k}} \dot{\gamma}^{\mathrm{k}} \mathbf{a}^{\mathrm{k}}
$$

Let us denote by $\mathbb{M}$ the elasticity tensor of the single crystal. The stress rate is given by :

$$
=\dot{\mathrm{p}} \mathbb{M}: \boldsymbol{\psi}-\sum_{1} \dot{\gamma}^{1} \mathbb{M}: \mathbf{a}^{1}
$$

Multiplying the two members of (23) by $\mathbf{a}^{\mathrm{k}}$ and denoting $\mathrm{T}^{\mathrm{k}}=\mathbf{a}^{\mathrm{k}}: \mathbb{M}: \boldsymbol{\Psi}$ and $\mathrm{B}^{\mathrm{kl}}=\mathbf{a}^{\mathrm{k}}: \mathbb{M}: \mathbf{a}^{1}$, we find :

$$
\dot{\tau}^{\mathrm{k}}=\dot{\mathrm{p}} \mathrm{T}^{\mathrm{k}}-\sum_{\mathrm{l}} \mathrm{B}^{\mathrm{kl}} \dot{\gamma}^{\mathrm{l}}
$$

Then, we find for $\dot{\mathrm{f}}^{\mathrm{k}}$ the following expression :

$$
\dot{\mathrm{f}} \mathrm{k}=\dot{\tau}^{\mathrm{k}}-\dot{\tau}_{\mathrm{c}}^{\mathrm{k}}=\dot{\mathrm{p}} \mathrm{T}^{\mathrm{k}}-\sum_{1} \mathrm{~B}^{\mathrm{kl}} \dot{\gamma}^{1}-\sum_{1} \mathrm{H}^{\mathrm{kl}} \dot{\gamma}^{1}
$$

or,

$$
\dot{\mathrm{f}}^{\mathrm{k}}=\dot{\mathrm{p}} \mathrm{T}^{\mathrm{k}}-\sum_{1} \mathrm{G}^{\mathrm{kl}} \dot{\gamma}^{1}
$$

with $\mathrm{G}^{\mathrm{kl}}=\mathrm{B}^{\mathrm{kl}}+\mathrm{H}^{\mathrm{kl}}$. If in this linear equations system all the rates are assumed to be $\mathrm{r}-\mathrm{h}$ rates, then, combined with the r-h flow rules (6), this system allows one to determine a solution of $\dot{\mathrm{f}}^{\mathrm{k}}$ and $\dot{\gamma}^{\mathrm{k}}$ for a given $\dot{\mathrm{p}}$.

The matrix $\mathrm{B}^{\mathrm{kl}}$ is constant as well as the factors $\mathrm{T}^{\mathrm{k}}$. Let us assume that the hardening matrix $\mathrm{H}^{\mathrm{kl}}$ is also constant (linear hardening). In this case, all the coefficients in the system (24) are constant. For a given increasing function of time $\mathrm{p}(\mathrm{t})$, the solution of 
these equations passes trough different stages in which different families of slip systems are active ( $k$ th system is active when $\mathrm{f}^{\mathrm{k}}=0, \dot{\mathrm{f}}^{\mathrm{k}}=0$ ). These families and the order of succession of different stages depend on the initial values of $\tau^{\mathrm{k}_{\mathrm{c}}}$ and the values of latent hardening coefficients $\mathrm{H}^{\mathrm{kl}}$. During a given stage, the solution of $\dot{\gamma}^{\mathrm{k}}$ is zero (if the system $\mathrm{k}$ is not active) or is a linear function of $\dot{\mathrm{p}}$ with constant coefficients. So the slope $\mathrm{d} \gamma^{\mathrm{k}} / \mathrm{dp}=\dot{\gamma}^{\mathrm{k}} / \dot{\mathrm{p}}$ is constant in a given stage. The solution $\gamma^{\mathrm{k}}$ as a function of $\mathrm{p}$ is therefore a piecewise linear function (figure 5.a). Slope discontinuities of the curves $\gamma^{k}(p)$ correspond to changes of active slip systems families. Let us notice that although $\gamma^{k}(p)$ are monotonous increasing functions, their slope can increase or decrease from one stage to the next depending on the values of the coefficients $G^{k l}$ and $T^{k}$ in the system (24).

Now we consider the stress component in the strain direction given by $q=\sigma: \psi$. The equation (23) leads to :

$$
\mathrm{dq} / \mathrm{dp}=\dot{\mathrm{q}} / \dot{\mathrm{p}}=\boldsymbol{\psi}: \mathbb{M}: \boldsymbol{\psi}-\sum_{\mathrm{k}} \mathrm{T}^{\mathrm{k}} \mathrm{d} \gamma^{\mathrm{k}} / \mathrm{dp}
$$

According to this equation, $\mathrm{q}$ like $\gamma^{\mathrm{k}}$ is a piecewise linear function of $\mathrm{p}$ (figure 5.b). But contrary to $\mathrm{d} \gamma^{\mathrm{k}} / \mathrm{dp}$, the slope $\mathrm{dq} / \mathrm{dp}$ is always a decreasing function of $\mathrm{p}$. Or, in other words, the slope discontinuities of the curve $q(p)$ which is piecewise linear, are all negative. This result has been established in the section 4.1 in the general case. It can be established directly for the case studied here too by applying the same general scheme of demonstration on the equations (21) to (25). It demands in particular to consider the rates in these equations, once as $r-h$ rates, obeying the r-h flow rules (6), and then as $1-h$ rates obeying 1 -h flow rules (7).

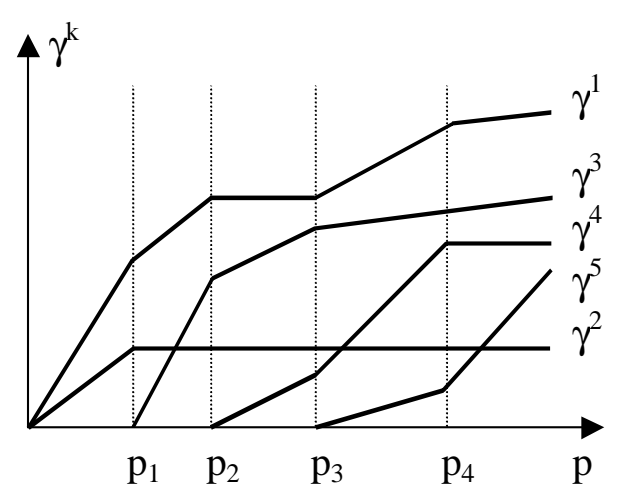

(a)

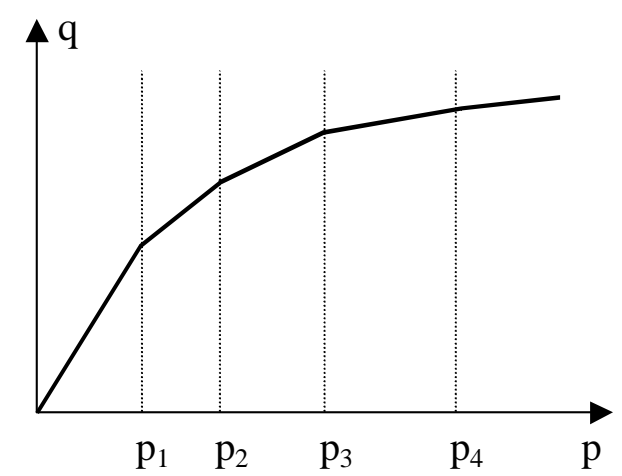

(b)

Figure 5 : Evolution of slip parameters $\gamma^{k}$ and of the stress q during a monotonous proportional straining path. Whereas the slope of the curves $\gamma^{k}$ can decrease or increase from one stage to the next, the slope of the stress curve is always decreasing.

This result, not mentioned in the works dealing with elastic-plastic modelling of single crystalline behaviour, has some consequences for phenomenological modelling of this 
behaviour. Consider for instance the hardening curve of fcc crystals (figure 6). The elastic stage is followed by a stage of linear hardening with a small slope called the easy glide (stage I on the figure 6). The end of this stage, is accompanied with an abrupt increase of the hardening modulus. Now, let us approximate this curve by a piecewise linear curve and consider the problem of phenomenological modelling for this behaviour by means of a model with a hardening matrix. An adequate expression for the elements of this matrix and an adequate set of initial values for critical shear stresses $\tau^{\mathrm{k}} \mathrm{c}$ must be found. The usual approximation of a constant hardening matrix leads, as seen above, to a piecewise linear strain-stress curve with a decreasing slope. This allows to model easily the two first stages of the hardening curve (elastic and easy glide). But the following stage can not be obtained by such a model. In fact, the rapid slope increase (at the end of the easy glide stage in fcc crystals) corresponds to a positive jump of slope in a piecewise linear approximation of the curve and it can not be obtained by a simple change of active slip systems family. This change leads, as shown above, leads to a negative jump.

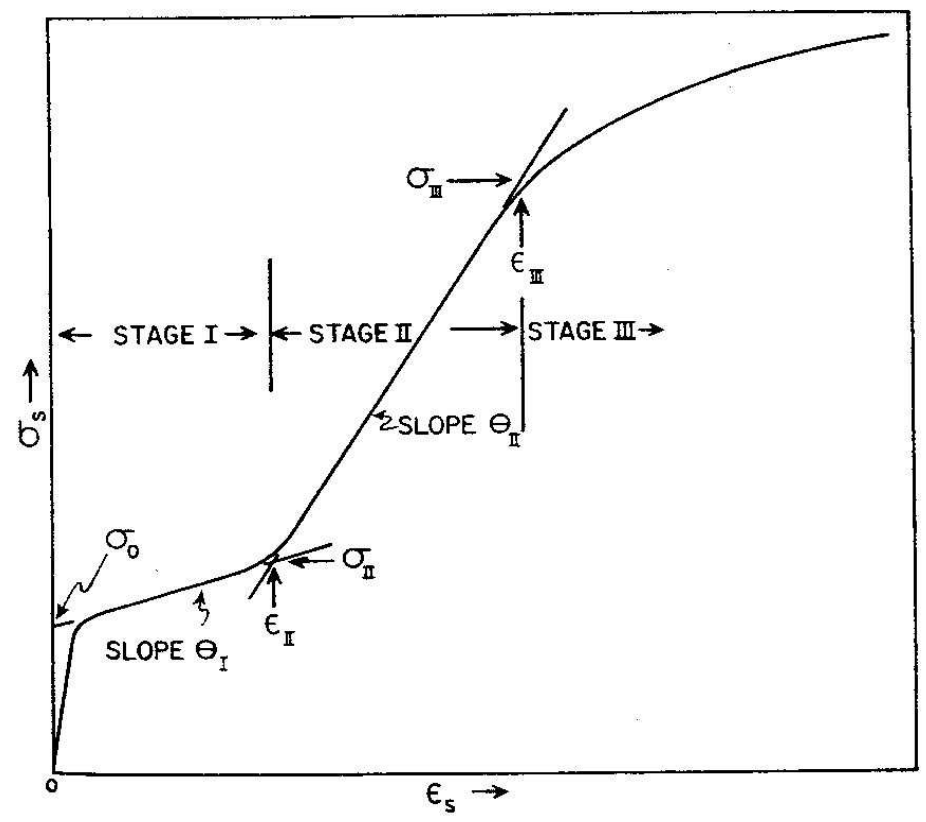

Figure 6 : Different stages of the hardening curves of fcc crystals : the stage of easy glide (stage I) ends on an abrupt change of slope (Davis and Gordon 1969).

It can be shown that even a slowly varying expression of the hardening matrix elements can not reproduce the rapid increase of slope at the end of the easy glide stage. To model this slope increase, a rapid variation, almost a rate jump, must be introduced in the expression of $\mathrm{H}^{\mathrm{kl}}$ as a function of the slip parameters $\left\{\gamma^{1}\right\}$. Now, it is interesting to see if the physical models of evolution of the coefficients $\mathrm{H}^{\mathrm{kl}}$ for fcc crystals are compatible 
with this rate jump. This means that one has to verify if the relationships between the latent hardening coefficients and the dislocation densities on different slip systems in fcc crystals give witness of the possibility of such a sharp variation. Only in this case, the models with a hardening matrix can be utilized to describe the behaviour of these crystals. Otherwise, these models are not adapted for these crystals, or, at least, some hypotheses adopted in these models leading to the inequality (18) must be abandoned or modified for these crystals. This can be the hypothesis of symmetry of the hardening matrix or the normality flow rule.

\subsection{Application to a micro-macro model of polycrystals}

In the framework of small strain theory, the self-consistent model of Berveiller and Zaoui (1978) for polycrystals establishes the following relation between the stress and strain tensors at the micro-level (grain, single crystal) and the macro-level ( homogenized polycrystal) :

$$
\sigma=\Sigma+2 \mu \alpha(1-\beta)\left(E^{\mathrm{p}}-\boldsymbol{\varepsilon}^{\mathrm{p}}\right)
$$

In this relation, $\boldsymbol{\sigma}$ and $\varepsilon^{\mathrm{p}}$ are respectively stress and plastic strain tensors at micro-level, and $\Sigma$ and $\mathrm{E}^{\mathrm{p}}$, the stress and strain tensors at macro-level. $\mu$ is the shear modulus and $\beta=2$ (4$5 v) /[15(1-v)]$ where $v$ is the Poisson's ratio. The macro-level strain and stress tensors are equal to the average of micro-level tensors in the polycrystal (average on all the grains of the polycrystal). This is written as :

$$
\mathrm{E}=\langle\boldsymbol{\varepsilon}\rangle, \quad \boldsymbol{\Sigma}=\langle\boldsymbol{\sigma}\rangle
$$

The parameter $\alpha$ is function a of the secant modulus of the hardening curve of the polycrystal for a monotonous proportional loading. If this parameter, in the relation (26), is taken constant and equal to 1, then one finds the Kröner (1961) model. The Kröner model is less accurate than Berveiller and Zaoui model, but leads to a much simpler calculation process. Indeed, the determination of $\alpha$ in the Berveiller and Zaoui model requires, at each load increment, an additional iteration loop which increases considerably the calculation time. However, for some polycrystals, in particular those constituted of NACl type crystals, it is possible to deduce the results of Berveiller and Zaoui model (for a monotonous proportional loading path) by a simple transformation of the results obtained by the Kröner model (Pouya 1991, 2000). This is possible for some types only of polycrystals, but, for these polycrystals, the simplification is very interesting. Nevertheless, to show that this transformation is valid, it is necessary to establish the following property :

"The secant modulus of the hardening curve of the polycrystal obtained with the Kröner model is a decreasing function of time under a monotonous proportional loading."

We try here to show this property using the results obtained on the stress and strain rate discontinuities. Since this property concerns the evolution of the secant modulus, its demonstration requires certainly information about the variation of stress and strain rates. Moreover, since the stress and strain rates of the polycrystal are the averages of those of their 
constitutive single crystalline grains, and since these rates can be discontinue, information about rate discontinuities is required to demonstrate this property.

It is easy to show that, for a monotonous increasing curve $q(p)$ which passes through the origin (figure 5), the secant modulus $q / p$ decreases if the slope $\mathrm{dq} / \mathrm{dp}$ decreases with $\mathrm{p}$. So, as to show the above property concerning the slope of the hardening curve, we try to show in the following that :

"The slope of the hardening curve of a polycrystal obtained with the Kröner model (for a monotonous proportional straining path) decreases with strain."

This property is not limited to $\mathrm{NaCl}$ polycrystals. It will be demonstrated for a general polycrystal under the assumption that the hardening matrices of the single crystalline grains are constant and positive, and it can be extended to some larger cases.

Now, to show this property, let suppose that the polycrystal is submitted to a monotonous proportional straining path given by :

$$
E=p \psi
$$

where $\mathrm{p}$ is a positive and increasing function of time and $\psi$ is a constant symmetric matrix. Let denote by $\mathbf{D}$ the deviator of $\boldsymbol{\psi}$ and by $\psi$ its trace : $\boldsymbol{\psi}=(\psi / 3) \boldsymbol{\delta}+\mathbf{D}$, where $\boldsymbol{\delta}$ is the unit tensor and $\mathbf{D}$ is traceless : $\operatorname{tr}(\mathbf{D})=0$.

Because the polycrystal is macroscopically isotropic, the plastic strain $\mathrm{E}^{\mathrm{p}}$ is proportional to D :

$$
\mathbf{E}^{\mathrm{p}}=\eta \mathbf{D}
$$

The elastic strain is then given by $\mathrm{E}^{\mathrm{e}}=\mathrm{E}-\mathrm{E}^{\mathrm{p}}$ and the stress by (linear isotropic elasticity) :

$$
\boldsymbol{\Sigma}=(\lambda+2 \mu / 3) \psi \text { p } \boldsymbol{\delta}+2 \mu \mathrm{g} \mathbf{D}
$$

where $\lambda$ and $\mu$ are the Lamé coefficients and :

$$
\mathrm{g}=\mathrm{p}-\eta
$$

The stress projection in the strain direction is :

We deduce from this relation that :

$$
\mathrm{q}=\Sigma: \psi=(\lambda+2 \mu / 3) \psi^{2} \mathrm{p}+2 \mu \mathrm{g} \mathbf{D}: \mathbf{D}
$$

where :

$$
\mathrm{dq} / \mathrm{dp}=(\lambda+2 \mu / 3) \psi^{2}+2 \mu \kappa \mathrm{dg} / \mathrm{dp}
$$

$$
\kappa=\mathbf{D}: \mathbf{D}
$$

According to this relation, dq/dp decreases with $\mathrm{p}$ if $\mathrm{dg} / \mathrm{dp}$ decreases with $\mathrm{p}$. To show that $\mathrm{dg} / \mathrm{dp}$ decreases, one could show that $\mathrm{d}^{2} \mathrm{~g} / \mathrm{dp}^{2}$ is negative if this second derivative existed. But, since the rates are discontinue, the second derivatives of different functions do not always exist. So, in the following, we try to show the decreasing of $\mathrm{dg} / \mathrm{dp}$ without calling to second derivatives. We precise that the first derivatives considered here are $\mathrm{r}-\mathrm{h}$ derivatives. This means for instance that $\mathrm{dg} / \mathrm{dp}=\dot{\mathrm{g}}^{+} / \dot{\mathrm{p}}^{+}$.

The relation (26), in which $\alpha$ is taken equal to 1 , can be written in the following form :

$$
\sigma+2 \mu(1-\beta) \varepsilon^{p}=\Sigma+2 \mu(1-\beta) E^{p}
$$

We deduce from this relation that $\tau$, the deviator of $\boldsymbol{\sigma}$ verifies : 


$$
\tau+2 \mu(1-\beta) \boldsymbol{\varepsilon}^{\mathrm{p}}=2 \mu[(1-\beta) \mathrm{p}+\beta \mathrm{g}] \mathbf{D}
$$

Now we consider an auxiliary single crystal having the same slip systems and the same critical shear stresses than the single crystalline grain in the relation (32), but with a shear modulus $\hat{\mu}=\mu(1-\beta)$. Let us suppose that this single crystal undergoes a monotonous proportional straining path given by :

with :

$$
\hat{\boldsymbol{\varepsilon}}=\xi \mathbf{D}
$$

$$
\xi=p+g \beta /(1-\beta)
$$

Since $\mathbf{D}$ is traceless, the stress in this crystal is given by $\hat{\boldsymbol{\sigma}}=2 \hat{\mu}\left(\hat{\boldsymbol{\varepsilon}}-\hat{\boldsymbol{\varepsilon}}^{\mathrm{p}}\right)$. This can be written as :

or :

$$
\hat{\boldsymbol{\sigma}}=2 \hat{\mu}\left(\xi \mathbf{D}-\hat{\boldsymbol{\varepsilon}}^{\mathrm{p}}\right)=2 \mu(1-\beta)\left\{[\mathrm{p}+\mathrm{g} \beta /(1-\beta)] \mathbf{D}-\hat{\boldsymbol{\varepsilon}}^{\mathrm{p}}\right\}
$$

$$
\hat{\boldsymbol{\sigma}}+2 \mu(1-\beta) \hat{\boldsymbol{\varepsilon}}^{\mathrm{p}}=2 \mu[(1-\beta) \mathrm{p}+\beta \mathrm{g}] \mathbf{D}
$$

This relation is identical to (32). On the other hand, $\hat{\boldsymbol{\sigma}}$ and $\hat{\boldsymbol{\varepsilon}}^{\mathrm{p}}$ in the auxiliary single crystal verify the same flow rules that the original single crystal because they have the same slip systems and critical shear stresses. So, $\tau$ and $\boldsymbol{\varepsilon}^{\mathrm{p}}$ are given by exactly the same equations than ${ }^{\wedge}$ and $\hat{\boldsymbol{\varepsilon}}$. This means that at every instant, $\tau=\hat{\boldsymbol{\sigma}}$ and $\boldsymbol{\varepsilon}^{\mathrm{p}}=\hat{\boldsymbol{\varepsilon}}^{\mathrm{p}}$.

The functions $\mathrm{g}$ and $\eta$ can be deduced from the average values of $\hat{\boldsymbol{\sigma}}$ and $\hat{\boldsymbol{\varepsilon}}^{\mathrm{p}}$ in the auxiliary grains. In fact, noticing that $\boldsymbol{\delta}: \mathbf{D}=0$, we deduce from (29) that :

On the other hand :

$$
\Sigma: \mathbf{D}=2 \mu \mathrm{gD}: \mathbf{D}=2 \mu \mathrm{kg}
$$

$$
\Sigma: \mathbf{D}=\langle\boldsymbol{\sigma}: \mathbf{D}\rangle=\langle\tau: \mathbf{D}\rangle=\langle\hat{\sigma}: \mathbf{D}\rangle
$$

We deduce from these relations that :

$$
\mathrm{g}=\frac{1}{2 \mu \kappa}\langle\hat{\sigma}: \mathbf{D}\rangle
$$

In the same way, we deduce from (28) that :

$$
\eta=\frac{1}{\kappa}\left\langle\hat{\boldsymbol{\varepsilon}}^{\mathrm{p}}: \mathbf{D}\right\rangle
$$

We will show first that $g$ and $\eta$ are increasing functions of $\xi$.

Let us consider provisionally the notations and equations of the section 4.2. Multiplying the two members of (24) by $\dot{\gamma}^{\mathrm{k}}$, and noticing that the flow rules imply that $\dot{\gamma}^{\mathrm{k}} \dot{\mathrm{f}}^{\mathrm{k}}=0$, one obtains, after summation upon $\mathrm{k}$ :

$$
\dot{\mathrm{p}} \sum_{\mathrm{k}} \dot{\gamma}^{\mathrm{k}} \mathrm{T}^{\mathrm{k}}=\sum_{\mathrm{k}} \sum_{1} \dot{\gamma}^{\mathrm{k}} \mathrm{G}^{\mathrm{kl}} \dot{\gamma}^{1}
$$

According to (21) and the definition of $\mathrm{T}^{\mathrm{k}}$, the first member of this relation is equal to $\dot{\mathrm{p}} \dot{\boldsymbol{\varepsilon}}^{\mathrm{P}}: \mathbb{\mathbb { N }}: \psi$. Since $\mathrm{G}^{\mathrm{kl}}=\mathrm{B}^{\mathrm{kl}}+\mathrm{H}^{\mathrm{kl}}$, and $\mathrm{B}^{\mathrm{kl}}$ is a positive matrix and $\mathrm{H}^{\mathrm{kl}}$ is assumed to be positive, then $G^{\mathrm{kl}}$ is a positive matrix. This means that the second member of (38) is 
always positive. So, $\dot{\mathrm{p}} \dot{\boldsymbol{\varepsilon}}^{\mathrm{P}}: \mathbb{\mathbb { V }}: \boldsymbol{\psi}$ is positive. In the same way one deduces from the relation :

$$
\dot{\mathrm{f}}^{\mathrm{k}}=\dot{\tau}^{\mathrm{k}}-\sum_{\mathrm{l}} \mathrm{H}^{\mathrm{kl}} \dot{\gamma}^{1}
$$

that :

$$
\sum_{\mathrm{k}} \dot{\gamma}^{\mathrm{k}} \dot{\tau}^{\mathrm{k}}=\sum_{\mathrm{k}} \sum_{\mathrm{l}} \dot{\gamma}^{\mathrm{k}} \mathrm{H}^{\mathrm{kl}} \dot{\gamma}^{1}
$$

According to (21) and the definition of $\tau^{\mathrm{k}}$, the first member of (39) is equal to $\dot{\boldsymbol{\sigma}}: \dot{\boldsymbol{\varepsilon}}^{\mathrm{P}}$, and, since $\mathrm{H}$ is a positive matrix, the second member is positive also. So ${ }^{\cdot}: \dot{\boldsymbol{\varepsilon}}^{\mathrm{P}}$ is positive and, since $\dot{\boldsymbol{\sigma}}: \dot{\boldsymbol{\varepsilon}}=\dot{\boldsymbol{\sigma}}: \dot{\boldsymbol{\varepsilon}}^{\mathrm{P}}+\dot{\boldsymbol{\varepsilon}}^{\mathrm{e}}: \mathbb{M}: \dot{\boldsymbol{\varepsilon}}^{\mathrm{e}}$, and $\dot{\boldsymbol{\varepsilon}}^{\mathrm{e}}: \mathbb{M}: \dot{\boldsymbol{\varepsilon}}^{\mathrm{e}} \geq 0$, then $\dot{\boldsymbol{\sigma}}: \dot{\boldsymbol{\varepsilon}}$ is also positive. Using the expression (22) of $\psi$, one deduces that $\dot{\mathrm{p}}^{\cdot}: \psi$ is positive.

The fact that, with the previous section notations, $\dot{\mathrm{p}} \dot{\boldsymbol{\varepsilon}}^{\mathrm{P}} \mathrm{\mathbb {M }}: \mathbf{\mathbb { V }}: \boldsymbol{\psi}$ and $\dot{\mathrm{p}} \dot{\sigma}: \boldsymbol{\psi}$ are positive, means that for the auxiliary single crystal considered in this section and submitted to the straining path (33), $\dot{\xi} \hat{\dot{\boldsymbol{\varepsilon}}}^{\mathrm{P}}: \mathbf{D}$ and $\dot{\xi} \hat{\dot{\sigma}}: \mathbf{D}$ are positive. The average of these expressions on the grains is then also positive. Using the relations (36) and (37), we find that $\dot{\xi} \dot{\eta}$ and $\dot{\xi} \dot{\mathrm{g}}$ are positive and this implies that $\dot{\eta} / \dot{\xi}$ and $\dot{\mathrm{g}} / \dot{\xi}$ are also positive. Since $\mathrm{d} \eta / \mathrm{d} \xi=\dot{\eta} / \dot{\xi}$ and $\mathrm{dg} / \mathrm{d} \xi=\dot{\mathrm{g}} / \dot{\xi}$, the derivatives $d \eta / d \xi$ and $d g / d \xi$ are positive. Now, let notice that the equations (30) and (32) imply :

and then :

$$
\mathrm{p}=(1-\beta) \xi+\beta \eta
$$

$$
\mathrm{dp} / \mathrm{d} \xi=1-\beta+\beta \mathrm{d} \eta / \mathrm{d} \xi
$$

Since $\beta, 1-\beta$ and $d \eta / d \xi$ are positives, this equation implies that that $d p / d \xi$ is positive. Then, noticing that :

we can deduce that $\mathrm{dg} / \mathrm{dp}$ is positive also.

$$
\mathrm{dg} / \mathrm{dp}=(\mathrm{dg} / \mathrm{d} \xi) /(\mathrm{dp} / \mathrm{d} \xi)
$$

The results concerning the rate discontinuities have not yet been used until this stage of demonstration in deal only with the sign of the first derivative of different functions. But they are indispensable in the following.

During a monotonous proportional straining path for the polycrystal, given by (27) with $\mathrm{p}$ increasing with time, $\xi$ also increases with time (because $\mathrm{dp} / \mathrm{d} \xi$ is positive), and, thus, each auxiliary grain of the polycrystal undergoes a monotonous proportional straining path (given by (33)). Denoting $\hat{q}=\hat{\boldsymbol{\sigma}}: \mathbf{D}$ and applying the results of the section 4.2 (based on the properties of rate discontinuities) to the auxiliary single crystal undergoing the monotonous proportional straining path (33), implies that $\mathrm{d} \hat{\mathrm{q}} / \mathrm{d} \xi$ is a decreasing function of $\xi$. Taking the average of $\mathrm{d} \hat{\mathrm{q}} / \mathrm{d} \xi$ on all the auxiliary grains and using (36), one deduces that $\mathrm{dg} / \mathrm{d} \xi$ is a decreasing function of $\xi$.

Let us now notice that the relation (32) leads to : 


$$
\mathrm{d} \xi / \mathrm{dp}=1+\frac{\beta}{1-\beta} \mathrm{dg} / \mathrm{dp}
$$

and then, using $d g / d p=(d g / d \xi)(d \xi / d p)$, we can write :

$$
\mathrm{dg} / \mathrm{dp}=(\mathrm{dg} / \mathrm{d} \xi)\left(1+\frac{\beta}{1-\beta} \mathrm{dg} / \mathrm{dp}\right)
$$

and deduce from this equation that :

$$
\mathrm{dg} / \mathrm{dp}=\frac{\mathrm{dg} / \mathrm{d} \xi}{1-\frac{\beta}{1-\beta} \mathrm{dg} / \mathrm{d} \xi}
$$

We showed above that $\mathrm{dg} / \mathrm{dp}$ and $\mathrm{dg} / \mathrm{d} \xi$ are positive, and then we deduce from (40) that the denominator $\left(1-\frac{\beta}{1-\beta} \mathrm{dg} / \mathrm{d} \xi\right)$ also is positive.

We showed above that $d g / d \xi$ decreases with $\xi$. Since $0<\beta<1, \frac{\beta}{1-\beta}$ is positive, and then (1$\frac{\beta}{1-\beta} d g / d \xi$ ) increases with $\xi$. Thus, in the second member of (40), the numerator and the denominator are both positive and the numerator decreases and the denominator increases with $\xi$. This implies that the fraction in the second member of (40) decreases with $\xi$. This means that $\mathrm{dg} / \mathrm{dp}$ decreases with $\xi$, and since, as showed above, $\xi$ increases with $\mathrm{p}$, we deduce that $\mathrm{dg} / \mathrm{dp}$ decreases with $\mathrm{p}$. The relation (31) implies then that dq/dp decreases with p.

The property announced here above concerning the deceasing character of the slope of the hardening curve of the polycrystal given by the Kröner model is, in this way, demonstrated using the results obtained for the stress an strain rate discontinuities.

\section{Comparison with other constitutive inequalities}

\subsection{Hill inequalities (normality flow rule)}

A bi-incremental version of the normality flow rule was given by Hill(1967, 1968a) in the following form:

$$
\dot{\mathbf{s}}^{+*}: \dot{\mathbf{e}}^{+\mathrm{e}}-\dot{\mathbf{s}}^{+\mathrm{e}}: \dot{\mathrm{e}}^{+*} \geq 0
$$

In this inequality, $\left(\dot{\mathbf{s}}^{+*}, \dot{\mathbf{e}}^{+*}\right)$ is a general $\mathrm{r}-\mathrm{h}$ admissible pair for a given state and $\left(\dot{\mathbf{s}}^{+\mathrm{e}}, \dot{\mathbf{e}}\right.$ ${ }^{+e}$ ) is an elastic r-h admissible pair for the same state. An elastic (i.e., reversible) r-h pair is a $\mathrm{r}$-h admissible pair which corresponds to a reversible process, and this means that the opposite pair is 1-h admissible (Figure 7.a). In other words, $\left(\dot{\mathbf{s}}^{+\mathrm{e}}, \dot{e}^{+\mathrm{e}}\right)$ is an elastic $\mathrm{r}-\mathrm{h}$ admissible pair if $\left(-\dot{\boldsymbol{s}}^{+\mathrm{e}},-\dot{\mathbf{e}}^{+\mathrm{e}}\right)$ is $1-\mathrm{h}$ admissible (and in this case $\left(-\dot{\mathbf{s}}^{+\mathrm{e}},-\dot{\mathbf{e}}^{+\mathrm{e}}\right)$ is an elastic $1-\mathrm{h}$ admissible pair). Applying the inequality (1) on the r-h admissible pair $\left(\dot{\mathbf{s}}^{+*}, \dot{\mathbf{e}}^{+*}\right)$ and the $1-\mathrm{h}$ 
admissible pair $\left(-\dot{\mathbf{s}}^{+\mathrm{e}},-\dot{\boldsymbol{e}}^{+\mathrm{e}}\right)$, one finds (41). So, the normality flow rule (41) is included in (1), or in other words, the inequality (1) generalizes the normality flow rule.

The figure (3) allows us to make a simple comparison between the inequalities (1) and (41). The first one is valid at all the points on the figure, whereas the second one, taking account of the fact that an elastic r-h rate $\left(\dot{\mathbf{s}}^{+\mathrm{e}}, \dot{\boldsymbol{e}}^{+\mathrm{e}}\right)$ is the opposite of an elastic l-h rate $\left(-\dot{\mathbf{s}}^{-\mathrm{e}}\right.$, $\left.-\dot{e}^{-\mathrm{e}}\right)$, characterizes the rates at the points $\mathrm{B}$ and $\mathrm{G}$ only .

\subsection{Petryk inequality for regular instants}

In the framework of models defined by the equations (2) to (6) and the hypothesis of symmetry of the hardening matrix, Petryk (1989) considers two r-h admissible pairs $\left(\dot{\mathbf{e}}^{+}, \dot{\mathbf{s}}^{+}\right)$ and $\left(\dot{\boldsymbol{e}}^{+*}, \dot{\mathbf{s}}^{+*}\right)$ for a given state, and shows that if the mechanisms active for the second pair (i.e. mechanisms $\mathrm{n}$ such that $\dot{\gamma}^{\mathrm{n+}}>0$ ) are non-unloaded for the first pair (i.e. $\dot{\mathrm{f}}^{\mathrm{n}+}=0$ ), then the following inequality is verified :

$$
\dot{\mathbf{s}}^{+}: \dot{\mathbf{e}}^{+^{*}}-\dot{\mathbf{s}}^{+^{*}}: \dot{\mathbf{e}}^{+} \geq 0
$$

This inequality gives rise to some interesting results concerning the macro-level behaviour of inhomogeneous elastic-plastic media (see further) with applications to stability and bifurcation study of these media (Petryk 1989) and possible extension to standard generalized materials (Nguyen and Petryk 1991). From this inequality, Petryk deduces another one :

$$
\dot{\mathbf{s}}^{+r}: \dot{\boldsymbol{e}}^{+^{*}}-\dot{\mathbf{s}}^{+^{*}}: \dot{\mathbf{e}}^{+r} \geq 0
$$

In this inequality, $\left(\dot{\mathbf{e}}^{+\mathrm{r}}, \dot{\mathbf{s}}^{+\mathrm{r}}\right)$ denotes the pair corresponding to the real straining process at a regular instant and $\left(\dot{\mathbf{e}}^{+*}, \dot{\mathbf{s}}^{+*}\right)$, a general $\mathrm{r}$-h admissible pair at this instant. A regular instant is defined as an instant for which all the rates are continuous.

Consider now a regular instant on a straining path and the real $1-\mathrm{h}$ and r-h rates $\left(\dot{\boldsymbol{e}}^{-\mathrm{r}}, \dot{\mathbf{s}}^{-\mathrm{r}}\right)$ and $\left(\dot{\boldsymbol{e}}^{+\mathrm{r}}, \dot{\mathbf{s}}^{+\mathrm{r}}\right)$ at this instant (Figure 7.b). These pairs of rates are necessarily l-h and $\mathrm{r}-\mathrm{h}$ admissible respectively, and moreover, by definition of the regular instant, $\left(\dot{\mathbf{e}}^{-\mathrm{r}}, \dot{\mathbf{s}}^{-}\right.$ $\left.{ }^{\mathrm{r}}\right)=\left(\dot{\boldsymbol{e}}^{+\mathrm{r}}, \dot{\mathbf{s}}^{+\mathrm{r}}\right)$. Applying the inequality (1) on the 1-h admissible pair $\left(\dot{\boldsymbol{e}}^{-\mathrm{r}}, \dot{\mathbf{s}}^{-\mathrm{r}}\right)$ and a general r$\mathrm{h}$ admissible pair $\left(\dot{\mathbf{e}}^{+*}, \dot{\mathbf{s}}^{+*}\right)$, one obtains (42). So, this inequality also is a particular case of the inequality (1), or in other words, the inequality (1) generalizes the Petryk inequality (42) for regular instants.

The figure (3) allows us once again a simple comparison between the two inequalities (1) and (42). Let consider the point $C$ on this figure. For this point it would be possible to continue the path $\mathrm{BC}$ without changing the direction, and this would define a regular path at the point $\mathrm{C}$. The «real » rates for this path at the point $\mathrm{C}$ would be the same that the 1 -h rates at this point for the path BC. Applying the inequality (42) to these «real regular» rates and the $r-h$ admissible rates corresponding to the path $C D$, would give the same result that the application of the inequality (1) at the point $C$ on the path BCD. So the inequality (42), with some extensions, allows us to obtain the results given by (1) for point $\mathrm{C}$, and in a general way for the points for which it is possible to continue the 1-h path in a continuous way. This is the case for the points $C, F$ and $G$ on the figure (3), but not for the points D and E : for these points the results given by (1) can not be deduced from (42). 


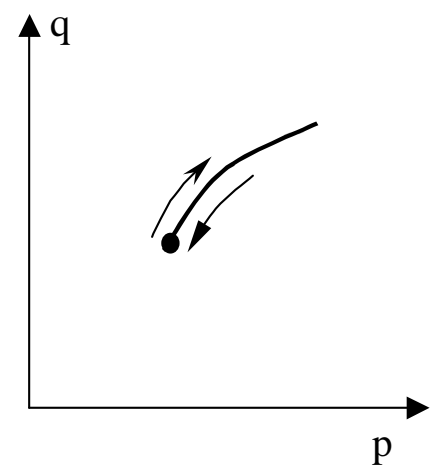

(a)

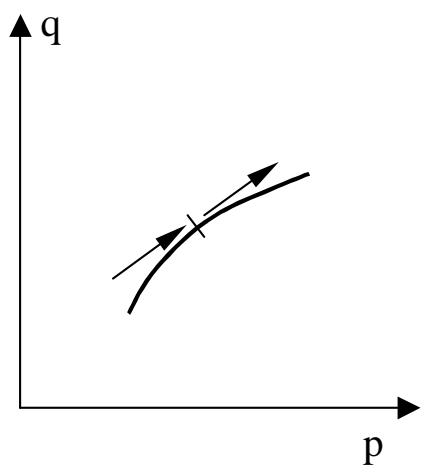

(b)

Figure 7 (a) : For an elastic (or reversible) deformation process, a right-hand admissible rate is the opposite of a left-hand admissible rate. (b): For a regular instant the real righthand rate is also left-hand admissible because equal to the real left-hand rate.

\section{Extension to macro-level for inhomogeneous materials}

Consider a inhomogeneous medium constituted at the micro-level of standard elasticplastic materials described above and which can be considered as homogeneous at some macroscopic scale. A macroscopic $\mathrm{r}-\mathrm{h}$ admissible pair $\left(\dot{\overline{\mathbf{e}}}^{+}, \dot{\overline{\mathbf{s}}}^{+}\right)$for this medium is defined as the average of a field of pairs $\left(\dot{\boldsymbol{e}}^{+}, \dot{\mathbf{s}}^{+}\right)$which are r-h admissible at every point and so that $\dot{\boldsymbol{e}}^{+}$ derives from a velocity filed, and $\dot{\boldsymbol{s}}^{+}$verifies local equilibrium equations. A macroscopic l-h admissible pair $\left(\dot{\overline{\mathbf{e}}}^{-}, \dot{\overline{\mathbf{s}}}^{-}\right)$is defined in the same way as an average of a field of locally 1-h admissible pairs. Then, an averaging theorem (Hill 1972) provides for these rates :

$$
\dot{\mathrm{s}}^{-}: \dot{\mathrm{e}}^{+}-\dot{\mathrm{s}}^{+}: \dot{\mathrm{e}}^{-}=\left\langle\dot{\mathrm{s}}^{-}: \dot{\mathrm{e}}^{+}-\dot{\mathrm{s}}^{+}: \dot{\mathrm{e}}^{-}\right\rangle
$$

where $\langle\cdot\rangle$ represents the average calculated over all material points. Since the local behaviour assures that $\dot{\mathbf{s}}^{-}: \dot{\mathbf{e}}^{+-} \dot{\mathbf{s}}^{+}: \dot{\mathbf{e}}^{-} \geq 0$ in every point, the average relation leads to :

$$
\dot{\overline{\mathbf{s}}}^{-}: \dot{\overline{\mathrm{e}}}^{+}-\dot{\overline{\mathbf{s}}}^{+}: \dot{\overline{\mathrm{e}}}^{-} \geq 0
$$

So, the inequality (1) is extended to macro-level. As in the case of micro-level inequalities, the inequality (43) generalizes a similar inequality given by Petryk (1989) and which can be written as :

$$
\dot{\overline{\mathbf{s}}}^{+\mathrm{r}}: \dot{\overline{\mathbf{e}}}^{+}-\dot{\overline{\mathbf{s}}}^{+}: \dot{\overline{\mathbf{e}}}^{\mathrm{r}} \geq 0
$$

In the inequality (44), obtained by the transmission of (42) to the macro-level, the pairs $\left(\dot{\overline{\mathbf{e}}}^{+\mathrm{r}}, \dot{\overline{\mathbf{s}}}^{+\mathrm{r}}\right)$ and $\left(\dot{\overline{\mathbf{e}}}^{+}, \dot{\overline{\mathbf{s}}}^{+}\right)$are $\mathrm{r}-\mathrm{h}$ admissible for a same state, and moreover $\left(\dot{\overline{\mathbf{e}}}^{+\mathrm{r}}, \dot{\overline{\mathbf{s}}}^{+\mathrm{r}}\right)$ must not 
induce abrupt unloading excepted in a zero volume region (Petryk 1989). So this inequality has a less general character than (43) in which the only condition imposed on the two pairs is to be respectively l-h and $\mathrm{r}-\mathrm{h}$ admissible for a same state.

\section{The constitutive inequality considered as a postulate}

In this section we will examine what will be the consequences of the inequality (1) if it is adopted as a postulate for elastic-plastic materials. First, reversible transformations will be considered and the symmetry of the elastic compliance tensor will be derived from this postulate. Then the normality flow rule will be deduced from this postulate. Finally, for models with an explicit hardening matrix, the local symmetry of this matrix will be examined. In consequence, it will be showed that the inequality (1), regarded as a postulate, synthesizes the three postulates of symmetry of the elastic tensor, normality flow rule and symmetry of the hardening matrix.

\subsection{Elastic transformations}

Let a state $\left(\mathbf{e}, \xi^{\mathrm{k}} ; \mathbf{s}\right)$ be given and for this state, let $\Phi^{-}$denote the set of 1-h admissible pairs $\left(\dot{\mathbf{e}}^{-}, \dot{\mathbf{s}}^{-}\right)$, and $\boldsymbol{\Phi}^{+}$the set of $\mathrm{r}-\mathrm{h}$ admissible pairs $\left(\dot{\mathbf{e}}^{+}, \dot{\mathbf{s}}^{+}\right)$. The sets $\boldsymbol{\Phi}^{-}$and $\boldsymbol{\Phi}^{+}$are subsets of the space $\mathrm{E} \times \mathrm{E}$ where $\mathrm{E}$ is the space of $3 \times 3$ symmetric second order tensors. Let also denote by $\tilde{\boldsymbol{\Phi}}^{-}$the set of pairs $\left(-\dot{\boldsymbol{e}}^{-},-\dot{\mathbf{s}}^{-}\right)$so that $\left(\dot{\mathbf{e}}^{-}, \dot{\mathbf{s}}^{-}\right) \in \boldsymbol{\Phi}^{-}$. Consider now an infinitesimal transformation leading from $\left(\mathbf{e}, \xi^{\mathrm{k}} ; \mathbf{s}\right)$ to a close neighbouring state $\left(\mathbf{e}^{\prime}, \xi^{\prime} \mathrm{k} ; \mathbf{s}^{\prime}\right)$ and let $\left(\dot{\mathbf{e}}^{+}, \dot{\mathbf{s}}^{+}\right)$be the pair of rates corresponding to this transformation. This pair is $\mathrm{r}-\mathrm{h}$ admissible for $\left(\mathbf{e}, \xi^{\mathrm{k}} ; \mathbf{s}\right)$. If the transformation is assumed to be reversible (so, theoretically, $\left.\xi^{\prime} \mathrm{k}=\xi^{\mathrm{k}}\right)$, then the transformation from $\left(\mathbf{e}^{\prime}, \xi^{\prime} \mathrm{k} ; \mathbf{s}^{\prime}\right)$ to $\left(\mathbf{e}, \xi^{\mathrm{k}} ; \mathbf{s}\right)$ is possible as well. In this case $\left(\mathbf{e}^{\prime}, \xi^{\prime} ; \mathbf{s}^{\prime}\right)$ is also a left-hand neighbour of $\left(\mathbf{e}, \xi^{\mathrm{k}} ; \mathbf{s}\right)$, what means that $\left(-\dot{\mathbf{e}}^{+},-\dot{\mathbf{s}}^{+}\right)$is $1-\mathrm{h}$ admissible for $\left(\mathbf{e}, \xi^{\mathrm{k}} ; \mathbf{s}\right)$, and so that $\left(\dot{\mathbf{e}}^{+}, \dot{\mathbf{s}}^{+}\right) \in \Phi^{+} \cap \tilde{\boldsymbol{\Phi}}^{-}$. The set of reversible or elastic rates pairs can then be defined as being the set $\boldsymbol{R}=\boldsymbol{\Phi}^{+} \cap \tilde{\boldsymbol{\Phi}}^{\text {. }}$.

Now let us examine the consequences of this definition.

Consider two general elements $(\dot{\boldsymbol{e}}, \dot{\mathbf{s}})$ and $\left(\dot{\boldsymbol{e}}^{*}, \dot{\mathbf{s}}^{*}\right)$ of $\boldsymbol{R}$. First, since $(\dot{\boldsymbol{e}}, \dot{\mathbf{s}}) \in \boldsymbol{\Phi}^{+}$and $\left(\dot{\mathbf{e}}^{*}, \dot{\mathbf{s}}^{*}\right) \in \tilde{\boldsymbol{\Phi}}^{-}$, what means that $\left(-\dot{\mathbf{e}}^{*},-\dot{\mathbf{s}}^{*}\right) \in \boldsymbol{\Phi}^{-}$, applying the inequality (1) one obtains $\dot{\mathbf{s}}^{*}: \dot{\mathbf{e}}-$ $\dot{\mathbf{s}}: \dot{\boldsymbol{e}}^{*} \leq 0$. Then, since $\left(\dot{\boldsymbol{e}}^{*}, \dot{\mathbf{s}}^{*}\right) \in \boldsymbol{\Phi}^{+}$and $(\dot{\boldsymbol{e}}, \dot{\mathbf{s}}) \in \widetilde{\boldsymbol{\Phi}}^{-}$, what means that $(-\dot{\mathbf{e}},-\dot{\mathbf{s}}) \in \boldsymbol{\Phi}^{-}$, applying the inequality (1) one obtains $\dot{\mathbf{s}}^{*}: \dot{\mathbf{e}}-\dot{\mathbf{s}}: \dot{\boldsymbol{e}}^{*} \geq 0$. This shows that $\dot{\boldsymbol{s}}^{*}: \dot{\boldsymbol{e}}-\dot{\mathbf{s}}: \dot{\boldsymbol{e}}^{*}=0$, and so :

$$
\forall(\dot{\mathbf{e}}, \dot{\mathbf{s}}),\left(\dot{\mathbf{e}}^{*}, \dot{\mathbf{s}}^{*}\right) \in \boldsymbol{R} ; \quad \dot{\mathbf{s}}^{*}: \dot{\mathbf{e}}-\dot{\mathbf{s}}: \dot{\mathbf{e}}^{*}=0
$$

If for a given state all infinitesimal transformations beginning from or ending to this state are reversible, then the inequality (1) for this state is converted to an equality. In usual terms such a state is situated within the elastic domain. 
Now, let denote respectively by $\boldsymbol{E}$ and $\boldsymbol{C}$ the sets of the first members and of the second members of the elements of $\boldsymbol{R}$. Every element of $\boldsymbol{R}$ is written as $\left(\dot{\boldsymbol{e}}^{+}, \dot{\boldsymbol{s}}^{+}\right)$where $\dot{\boldsymbol{e}}^{+} \in \boldsymbol{E}$ and $\dot{\mathbf{s}}^{+} \in \boldsymbol{C}$. Let now assume that $\boldsymbol{C}$ is not empty, and that it contains 6 linearly independent elements of the space E. This topological hypothesis is made to exclude some pathologic cases of elastic domain, for instance, the case where this domain in the principle stress space (dimension 3 ) would be a surface ( 2 dimensional variety) instead of a volume (3 dimensional variety).

With this hypothesis, the equality (45) allows us to demonstrate that there exists a unique fourth order tensor $\mathbb{M}$ having the symmetry $M_{i j k l}=M_{j i k l}=M_{k l i j}$ verifying :

$$
\forall(\dot{\mathbf{e}}, \dot{\mathbf{s}}) \in \boldsymbol{R}, \quad \dot{\mathbf{e}}=\mathbb{M}: \dot{\mathbf{s}}
$$

In fact, consider a family of six linearly independent stress rates $\dot{\mathbf{s}}_{1}, \ldots, \dot{\mathbf{s}}_{6} \in \boldsymbol{C}$ and the pairs $\left(\dot{\boldsymbol{e}}_{1}, \dot{\mathbf{s}}_{1}\right), \ldots,\left(\dot{\mathbf{e}}_{6}, \dot{\mathbf{s}}_{6}\right) \in \boldsymbol{R}$. At this stage of demonstration the choice of $\dot{\boldsymbol{e}}_{\mathrm{k}}$ for $\dot{\boldsymbol{s}}_{\mathrm{k}}$ is not necessary unique, but let be fixed a family of $\dot{\boldsymbol{e}}_{\mathrm{k}}$. For a general $\dot{\mathbf{s}} \in \boldsymbol{C}$, and a pair $(\dot{\boldsymbol{e}}, \dot{\mathbf{s}}) \in \boldsymbol{R}$ the equality (45) provides :

$$
\forall \mathrm{k} \in\{1, \ldots, 6\} ; \quad \dot{\mathbf{s}}_{\mathrm{k}}: \dot{\mathbf{e}}=\dot{\mathbf{s}}: \dot{\mathbf{e}}_{\mathrm{k}}
$$

Then, since $\dot{\boldsymbol{s}}_{\mathrm{k}}$ are linearly independent, this determines for $\dot{\boldsymbol{s}}$ a unique $\dot{\boldsymbol{e}}$ as a linear function of $\dot{\boldsymbol{s}}$. This function can be written as (46). The symmetries $M_{i j k l}=M_{j i k l}=M_{i j l k}$ are trivially due the symmetries of $\dot{\mathbf{e}}, \dot{\mathbf{s}}$ as second order tensors. The diagonal symmetry $M_{i j k l}=M_{k l i j}$ is a non trivial result, and can be deduced from the following equality resulting from (45) and (46) :

$$
\forall \mathrm{p}, \mathrm{q} \in\{1, \ldots, 6\}, \dot{\mathbf{s}}_{\mathrm{p}}: \mathbb{M}: \dot{\mathbf{s}}_{\mathrm{q}}=\dot{\mathbf{s}}_{\mathrm{q}}: \mathbb{M}: \dot{\mathbf{s}}_{\mathrm{p}}
$$

It may be remarked that if the hypothesis $\boldsymbol{C}$ containing 6 linearly independent elements of $E$ is not satisfied, then the solution for $\mathbb{M}[$ is not unique, but its restriction to the subspace of E generated by the elements of $\boldsymbol{C}$ will be unique and symmetric.

\subsection{Normality flow rule}

As shown in (\$5.1), the normality flow rule (41) can be deduced from the inequality (1). In the context of finite strain, this expression of the normality rule has the measure invariance property and is preferable to the earlier expression calling to a convex elastic domain (Hill 1968b). A variant version of this normality rule was given by Hill (1972), Hill and Rice (1972) and Hill and Rice (1973) in the following form :

$$
\dot{\mathbf{e}}^{\mathrm{p}+*}: \dot{\mathbf{s}}^{\mathrm{e}+} \leq 0
$$

In this inequality $\dot{\boldsymbol{e}}^{\mathrm{p}+*}$ is a general plastic strain rate, and $\dot{\boldsymbol{s}}^{\mathrm{e}+}$ is the stress rate corresponding to an elastic unloading. The plastic strain rate for a pair $(\dot{\mathbf{e}}, \dot{\mathbf{s}})$ is defined as $\dot{\mathbf{e}}^{\mathrm{p}}=\dot{\mathbf{e}}-\mathbb{M}: \dot{\mathbf{s}}$. If the inequality (41) is written for the pair $\left(\dot{\mathbf{e}}^{\mathrm{e}+}, \dot{\mathbf{s}}^{\mathrm{e}+}\right)$ and the pair $\left(\dot{\boldsymbol{e}}^{+*}, \dot{\mathbf{s}}^{+*}\right)$, with substituting $\dot{\mathbf{e}}^{\mathrm{p}{ }^{+*}}=\dot{\mathbf{e}}^{+^{*}}-\mathbb{M}: \dot{\mathbf{s}}^{+^{*}}$ and $\dot{\mathbf{e}}^{\mathrm{e}+}=\mathbb{M}: \dot{\mathbf{s}}^{\mathrm{e}+}$, and using the diagonals symmetry of 
$\mathbb{M}$, then (47) will be obtained. So, this version of the normality flow rule also is well a consequence of the postulate (1).

The normality rule (47) can be completed by the postulate (1) for 1-h plastic rates. This can be down by taking $\left(\dot{\boldsymbol{e}}^{+\mathrm{e}}, \dot{\mathbf{s}}^{+\mathrm{e}}\right) \in \boldsymbol{R}$ and applying (1) on this $\mathrm{r}-\mathrm{h}$ admissible pair and a general l-h admissible pair $\left(\dot{\boldsymbol{e}}^{-*}, \dot{\mathbf{s}}^{-*}\right)$. Then if $\dot{\mathbf{e}}^{-*}$ is substituted by $\dot{\mathbf{e}}^{-*}=\dot{\mathbf{e}}^{\mathrm{p}-*}+\mathbb{M}: \dot{\mathbf{s}}^{-*}$, one obtains : $\dot{\mathbf{e}}^{\mathrm{p}+*}: \dot{\mathbf{s}}^{+\mathrm{e}} \leq 0$. The completed version of the normality rule (47) can be written then as the following:

$$
\begin{aligned}
& \forall \dot{\mathbf{s}}^{+} \in C, \quad \dot{\mathrm{e}}^{\mathrm{p}+^{*}}:\left(-\dot{\mathbf{s}}^{+}\right) \geq 0 \\
& \forall \dot{\mathbf{s}}^{+} \in \boldsymbol{C}, \quad \dot{\mathrm{e}}^{\mathrm{p}^{-*}}:\left(-\dot{\mathbf{s}}^{+}\right) \geq 0
\end{aligned}
$$

\subsection{Local symmetry of the hardening matrix}

The framework of elastic-plastic models with explicit hardening matrix (equations 2 to 7) is considered and it is assumed that the $\gamma^{\mathrm{k}}$ constitute an independent family of variables (normal internal variables). This means that the $\dot{\gamma}^{\mathrm{k}}$ of potentially active mechanisms can take independent positive values. Let consider two potentially active mechanisms denoted by 1 and 2 , and try to show that $\mathrm{H}^{12}=\mathrm{H}^{21}$. Consider the $1-\mathrm{h}$ and $\mathrm{r}-\mathrm{h}$ straining paths for which these mechanisms are the only $1-h$ and r-h active mechanisms, this means that all the $\dot{\gamma}^{k-}$ and $\dot{\gamma}^{k-}$ are equal to zero except for $\dot{\gamma}^{1-}, \dot{\gamma}^{2-}, \dot{\gamma}^{1+}, \dot{\gamma}^{2+}$ (this is possible because of the independence of the mechanisms). According to the relation (3), the $1-\mathrm{h}$ and $\mathrm{r}-\mathrm{h}$ plastic strain rates will be given respectively by :

and :

$$
\dot{\mathbf{e}}^{\mathrm{p}-}=\dot{\gamma}^{1-}\left(\partial \mathrm{f}^{1} / \partial \mathbf{s}\right)+\dot{\gamma}^{2-}\left(\partial \mathrm{f}^{2} / \partial \mathbf{s}\right)
$$

$$
\dot{\mathbf{e}}^{\mathrm{p}+}=\dot{\gamma}^{1+}\left(\partial \mathrm{f}^{1} / \partial \mathbf{s}\right)+\dot{\gamma}^{2+}\left(\partial \mathrm{f}^{2} / \partial \mathbf{s}\right)
$$

All the expressions $\Delta_{\mathrm{n}}$ in the inequality (8) vanish since $\dot{\mathrm{f}}^{1-}=\dot{\mathrm{f}}^{2-}=\dot{\mathrm{f}}^{1+}=\dot{\mathrm{f}}^{2+}=0$ and $\dot{\gamma}^{\mathrm{k}-}=$ $\dot{\gamma}^{\mathrm{k}+}=0$ for $\mathrm{k} \neq 1$ and $\mathrm{k} \neq 2$, and one finds :

$$
\dot{\mathbf{e}}^{\mathrm{p}+}: \dot{\mathbf{s}}^{-}-\dot{\mathbf{e}}^{\mathrm{p}-}: \dot{\mathbf{s}}^{+}=\left(\mathrm{H}^{12}-\mathrm{H}^{21}\right)\left(\dot{\gamma}^{1+} \dot{\gamma}^{2-}-\dot{\gamma}^{1-} \dot{\gamma}^{2+}\right)
$$

The postulate (1) and the diagonal symmetry of $M$ (which is now a consequence of this postulate), allow us to establish the inequality (9). This inequality and the relation (50) lead then to :

$$
\left(\mathrm{H}^{12}-\mathrm{H}^{21}\right)\left(\dot{\gamma}^{1+} \dot{\gamma}^{2-}-\dot{\gamma}^{1-} \dot{\gamma}^{2+}\right) \geq 0
$$

This inequality must be verified for arbitrary positive values of $\dot{\gamma}^{1-}, \dot{\gamma}^{1+}, \dot{\gamma}^{2-}, \dot{\gamma}^{2+}$. This is possible only if $\mathrm{H}^{2}-\mathrm{H}^{21}=0$. This shows the symmetry of the hardening matrix concerning the mechanisms 1 and 2 .

In this way, the symmetry of all the cross terms of the hardening matrix corresponding to pairs of mechanisms which can be simultaneously potentially active, i.e., its local symmetry, is demonstrated. 


\subsection{Equivalence of postulates}

Let denote the postulate of symmetry of the elastic compliance tensor by $\mathrm{P}_{1}$, the normality flow rule by $\mathrm{P}_{2}$, and the local symmetry of the hardening matrix (in the framework of models with a hardening matrix and with normal internal variables) by $\mathrm{P}_{3}$. Then, the essential result of demonstrations given above is that, the postulate (1) is equivalent to the sum of these three postulates, hat can be written as :

$$
\{\text { Inequality (1) }\} \Leftrightarrow\left\{\mathrm{P}_{1}, \mathrm{P}_{2}, \mathrm{P}_{3}\right\}
$$

So, the inequality (1) considered as a postulate, defines the same elastic-plastic behaviour than these three postulates.

\section{Conclusion}

The originality of the inequality (1) consists in involving the left-hand rates. This allows us a full integration of these rates in the study of elastic-plastic behavior. As shown here above, this enables one to obtain some original results concerning the rate discontinuities in elastic-plastic transformation processes. This allows us also to generalize some other important constitutive inequalities like the Hill's normality flow rule (41) and the Petryk inequalities (42) and (44). This probably also provides a tool for investigation of retrograde problems which have not been well studied in plasticity.

The domain of application of this inequality is not restricted to elastic-plastic materials. In fact, the general standard materials for which this inequality was established here above, include some families of damaging materials. The validity of this inequality is likely extendible to a large family of time-independent dissipative materials. 


\section{References}

Berveiller M., Zaoui A., 1978. An extension of the self-consistant scheme to plasticity flowing polycrystals. J. Mech. Phys. Solids 26, pp. 325-344.

Brünig M, 2002. Numerical analysis and elastic ${ }^{-}$plastic deformation behavior of anisotropically damaged solids. Int. J Plasticity 18 (9) 1237-1270.

Brünig M., 2003. An anisotropic ductile damage model based on irreversible thermodynamics. Int J Plasticity (Article in Press).

Budiansky B., Wu T.T., 1962. Theoretical prediction of plastic strains of polycrystals. In : Proc. 4th Cong. Appl. Mech., pp. 1175-1185.

Chen S. H. and Wang T. C. 2002. A new deformation theory with strain gradient effects. Int. J Plasticity 18 (8), 971-995.

Chiarellia, A.S., Shao J. F., Hoteit N., 2003. Modeling of elastoplastic damage behavior of a claystone. Int. J Plasticity 19 (1) 23-45.

Chun B. K., Jinna J. T. and Lee J. K., 2002. Modeling the Bauschinger effect for sheet metals, part I : theory. Int. J Plasticity 18 (5-6) 571-595.

Cleja-Tigoiu S., 2003. Consequences of the dissipative restrictions in finite anisotropic elasto-plasticity. Int J Plasticity, Volume 19, Issue 11, November 2003, Pages 1917-1964 Davis, L.A., Gordon, R.B., 1969. Plastic deformation of alkali halide crystals at high pressure: work-hardening effects. J. Appl. Phys., vol. 40, N. 11, october 1969, pp. 45074513.

Drozdov A. D. and Dorfmann Al., 2002. A micro-mechanical model for the response of filled elastomers at finite strains. Int. J Plasticity 19 (7) 1037-1067.

Drucker, D.C., 1951. A more fundamental approach to plastic stress-strain rate relations. In : Proc. First US Congress of Applied Mechanics, ed. ASME (ASME New York, pp. 487491.

Drucker, D.C., 1959. A definition of stable inelastic material. ASME J. Appl. Mech. 26, pp. 101-106.

Gupta V., Bergström J. S., 2002. A progressive damage model for failure by shear faulting in polycrystalline ice under biaxial compression. Int. J Plasticity 18 (4) 507-530.

Gurtin M.E., 2003. On a framework for small-deformation viscoplasticity: free energy, microforces, strain gradients. Int. J Plasticity 19 (1) 47-90.

Halphen, B., Nguyen, Q.S., 1975. Sur les matériaux standards généralisés. J. Méc. 14, pp. 39-63.

Haupt P. and Kersten Th., 2003. On the Modelling of Anisotropic Material Behaviour in Viscoplasticity. Int J Plasticity, Volume 19, Issue 11, November 2003, Pages 1885-1915.

Henry W. and Haslach Jr., 2002. A non-equilibrium thermodynamic geometric structure for thermoviscoplasticity with maximum dissipation. Int J Plasticity 18 (2) 127-153.

Hill, R., 1966. Generalized constitutive relations for incremental deformation of metal crystals by multislip. J. Mech. Phys. Solids 14,pp. 95-102. 
Hill, R., 1967. The essential structure of constitutive laws for metal composites and polycrystals. J. Mech. Phys. Solids 15, pp. 79-95.

Hill, R., 1968a. On constitutive inequalities for simple materials-I. J. Mech. Phys. Solids 16, pp. 229-242.

Hill, R., 1968b. On constitutive inequalities for simple materials-II. J. Mech. Phys. Solids 16, pp.315-322.

Hill, R., 1972. On constitutive macro-variables for heterogeneous solids at finite strain. In : Proc. R. Soc. Lond. A326, p. 121.

Hill, R., Rice, J.R., 1972. Constitutive analysis of elastic-plastic crystals at arbitrary strain. J. Mech. Phys. Solids 20, pp. 401-413.

Hill, R., Rice, J.R., 1973. Elastic potentials and the structure of inelastic constitutive laws. SIAM J. Appl. Math. 25, pp. 448.

Houlsby and Purzin 2000. A thermodynamical framework for constitutive models for rateindependent dissipative materials. Int J Plasticity 16, 1017-1047.

Iliushin, A.A., 1961. On the postulate of stability. J. Appl. Math. Mech. 25, (1961) pp. 746752.

Kaczmarek J., 2003. A nanoscale model of crystal plasticity. Int J Plasticity Volume 19, Issue 10, October 2003, Pages 1585-1603.

Kang G., Ohno N. and Nebu A., 2003. Constitutive Modeling of Strain Range Dependent Cyclic Hardening. Int J Plasticity Volume 19, Issue 10, October 2003, Pages 1801-1819.

Koiter, W.T., 1960. General theorems for elastic-plastic solids. In: Progress in solid mechanics, vol.1, North Holland Publishing Company, Amsterdam.

Krempl E. and Khan F., 2003. Rate (time)-dependent deformation behavior: an overview of some properties of metals and solid polymers. Int. J Plasticity 19 (7) 1069-1095.

Kröner, E., 1961. Zur Plastischen Verformung des Viel-Kristalls. Acta. Met. 9, p. 155.

Langlois L. and Berveiller M., 2003. Overall softening and anisotropy related with the formation and evolution of dislocation cell structures. Int. J Plasticity 19 (5) 599-624.

Li S., Hoferlina E., Van Baela A., Van Houttea P. and Teodosiub C., 2003. Finite element modeling of plastic anisotropy induced by texture and strain-path change. Int. J Plasticity 19 (5) 647-674.

Mandel, J., 1965. Généralisation de la théorie de plasticité de W.T. Koiter. Int. J. Solids Structures 1, pp.273-295.

Marigo J.J., 1989. Constitutive relations in plasticity, damage and fracture mechanics based on a work property. Nuclear Engineering and Design 114, pp. 249-272.

Naboulsi S. K. and Palazotto A. N., 2003. Damage model for metal-matrix composite under high intensity loading. Int. J Plasticity 19 (4) 435-468.

Nguyen, Q.S., Petryk, H., 1991. A constitutive inequality for time-independent dissipative solids. C.R. Acad. Sci. Paris, t.312, Série II, pp. 7-12.

Petryk, H., 1989. On constitutive inequalities and bifurcation in elastic-plastic solids with a yield-surface vertex. J. Mech. Phys. Solids 37, pp.265-291.

Pouya, A., 1991. Comportement rhéologique du sel gemme, application à l'étude des excavations souterraines. PhD Thesis, Ecole Nationale des Ponts et Chaussées, Paris. 
Pouya, A., 1993. Deformation of multipotential elastic-plastic solids : inequalities verified by stress and strain rate discontinuities. C.R. Acad. Sci. Paris, t.316, Série II, pp. 15111516.

Pouya, A., 2000. A micro-macro approach for rock salt behaviour. Eur. J. Mech. A/Solids 19, pp. 1015-1028.

Rice, J.R., 1971. Inelastic constitutive relations for solids: An internal-variable theory and its application to metal plasticity. J. Mech. Phys. Solids 19, pp. 433-455.

Rajagopal K.R. and Srinivasa A.R. 1998a. Inelastic behaviour of materials - Part 1, theoretical underpinnings. Int. J Plasticity 14 (10-11), 945-967.

Rajagopal K.R. and Srinivasa A.R. 1998b. Inelastic behaviour of materials - Part 1, inelastic response. Int. J Plasticity 14 (10-11), 969-995.

Scheidler M. and Wright T. W., 2003. Classes of flow rules for finite viscoplasticity. Int. J Plasticity 19 (8) 1119-1165.

Stoughton Th. B., 2002. A non-associated flow rule for sheet metal forming. Int. J Plasticity 18 (5-6) 687-714.

Taleb L. and Sidoroff F., 2003. A micromechanical modelling of the Greenwood-Johnson mechanism in transformation induced plasticity. Int J Plasticity (Article in Press).

Taylora M. B., Zbib H. M. and Khaleel M. A., 2002. Damage and size effect during superplastic deformation. Int. J Plasticity 18 (3), 414-442.

Zhang, T., Lee, J., 2003. A plasticity model for cellular materials with open-celled structure. Int. J Plasticity 19 (6) 749-770.

Van Houtte P., Delannaya L. and S. R. Kalidindib, 2002. Comparison of two grain interaction models for polycrystal plasticity and deformation texture prediction. Int. $\mathrm{J}$ Plasticity 18 (3), 359-377.

Yoshida F. and Uemori T., 2002. A model of large-strain cyclic plasticity describing the Bauschinger effect and work hardening stagnation. Int. J Plasticity 18 (5-6) 661-686.

Zhou Z.D., Zhao S.X. and Kuang Z.B. 2003. An integral elasto-plastic constitutive theory. Int J Plasticity, Volume 19, Issue 9, September 2003, Pages 1377-1400.

Ziegler, H., 1983. An Introduction to Thermodynamics, First Edition, McGraw, London. 